\title{
Flow around a scoured bridge pier: a stereoscopic PIV analysis
}

\author{
Ulrich Jenssen ${ }^{1} \cdot$ Michael Manhart ${ }^{1}$ (1)
}

Received: 3 February 2020 / Revised: 15 July 2020 / Accepted: 29 August 2020 / Published online: 22 September 2020

(c) The Author(s) 2020

\begin{abstract}
We performed stereoscopic particle image velocimetry of the turbulent flow inside a scour hole around a cylinder in a sandy bed. At two planes, symmetry plane and $45^{\circ}$ with respect to the approach flow, the flow and its turbulence structure were investigated. We used two Reynolds numbers $(20,000$ and 39,000) based on the cylinder diameter and the depth-averaged velocity in the symmetry plane. The flow is characterized by a strong down-flow in front of the cylinder, a large horseshoe vortex inside the scour, and an upstream directed wall jet underneath. The values of vorticity in the horseshoe vortex and of the velocity in the wall jet are larger than in a comparable configuration on a flat bed. Enhanced levels of turbulent kinetic energy are found around the horseshoe vortex and in the shear layer detaching from the rim. The orientation of the main axis of the velocity fluctuations changes when the flow enters the scour hole: from about wall-parallel in the detaching shear layer to vertical at the horseshoe vortex. The production of turbulent kinetic energy shows a maximum upstream of the horseshoe vortex centre with considerable production in the shear layer and in the wall jet underneath the horseshoe vortex. Furthermore, strong wall-parallel velocity fluctuations are visible in this region, and bimodal velocity distributions are found, but not anywhere else. The time-averaged wall-shear stresses are largest under the horseshoe vortex and most likely larger than in a corresponding flat-bed configuration.
\end{abstract}

\section{Graphic abstract}

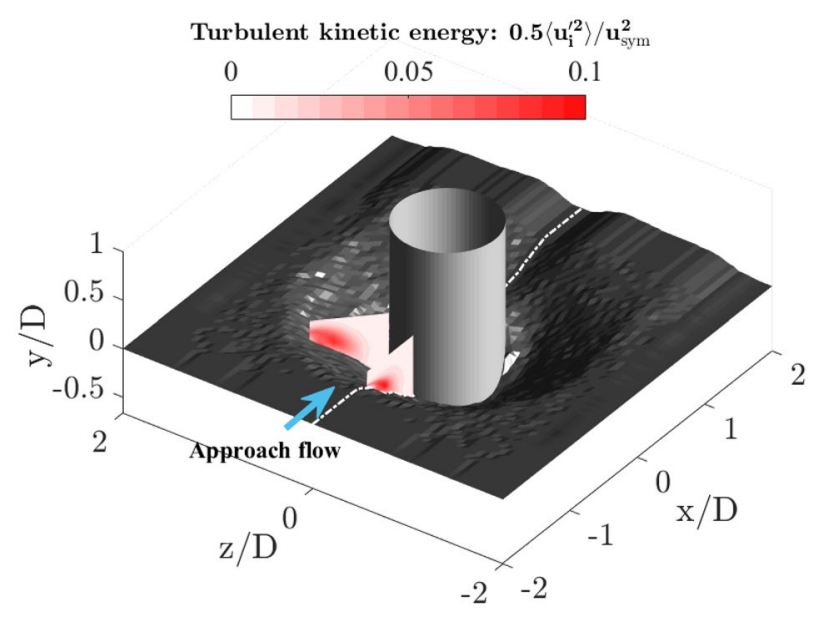

Michael Manhart

michael.manhart@tum.de

1 Hydromechanics, Technical University of Munich, Arcisstr. 21, 80333 Munich, Germany

\section{Introduction}

Local disturbances of the flow around hydraulic structures such as bridge piers can result in enhanced wall-shear stress levels and the development of local scour holes. Such scour holes can be a severe threat for the safety of the hydraulic structure. The estimation of the depth of a scour hole is a 
difficult task and empirical formulae for the design of pier foundations go in hand with large uncertainties, e.g., (Link et al. 2008).

The coupling of a detailed numerical simulation with a model for sediment transport and/or erosion could considerably reduce these uncertainties as Roulund et al. (2005) pointed out. They investigated the scour development in front of a cylinder by a combined experimental/numerical study. Although the sediment transport model was calibrated, they observed a discrepancy of about $15 \%$ in the scour depth upstream the cylinder and assigned this deviation to the numerical method to model the erosion process. Similar difficulties have been observed by Khosronejad et al. (2012). They attribute the difficulties in finding a correct scour shape and growth rate to the uncertainties in the turbulence and sediment transport models. A first principle prediction of scour development without calibration seems to be still out of reach, as uncertainties are not only persistent in current erosion and sediment transport models but also in current flow simulation methods. There are many sources of errors, and a deep understanding of the flow field is required for a detailed and reliable scour modelling.

The flow around a bridge pier (circular cylinder) is governed by the down-flow generated by the approaching boundary layer (Unger and Hager 2007). This down-flow entails fluid with high momentum towards the bottom wall deflecting the flow, such that the so-called horseshoe vortex occurs (Baker 1980). While the flow passes the cylinder, the wall-shear stress strongly increases at the side of the cylinder (Melville 1975) initiating the erosion of bed material. As the scour hole gets deeper, it moves towards the cylinder front. The scour hole evolves until an equilibrium state is reached in which the forces from the flow on the sediment grains decreased below the critical threshold.

While the scour hole develops around the cylinder, the horseshoe vortex is always present. The strength of this vortex is largest in the symmetry plane upstream of the cylinder (Melville and Raudkivi 1977; Dey and Raikar 2007), while the vortex weakens when bending around the cylinder. This indicates that the horseshoe vortex is driven by the downflow at the cylinder front, which, in turn, is dependent on the approaching flow conditions (Unger and Hager 2007; Schanderl and Manhart 2016). In the initial phase of the scour (i.e., cylinder on flat bed), the magnitude of the downflow is approximately $0.4 u_{\mathrm{b}}$ (Schanderl et al. 2017), with $u_{\mathrm{b}}$ as the depth-averaged velocity of the approaching flow. While the scour reaches its equilibrium state, the down-flow increases up to approximately $0.7 u_{\mathrm{b}}$ (Melville and Raudkivi 1977; Graf and Istiarto 2002; Dey and Raikar 2007; Unger and Hager 2007) as it can accelerate over a longer distance along the cylinder front.

Since the angle of inclination of the scour surface was observed to be larger than the angle of repose of the sediment, e.g., Melville and Raudkivi (1977); Link et al. (2008), it is generally accepted that the rotating horseshoe vortex stabilises the scour slope. Dey and Raikar (2007) observed that the horseshoe vortex in the scour hole has an elliptical shape with an orientation parallel to the surface of the scour. Furthermore, they document a "reversed flow along the sloping bed" with a magnitude of $0.2 u_{\mathrm{b}}$, which might be a hint for the existence of a near-wall jet similar to the one observed by Devenport and Simpson (1990) in the flat-bed case.

The boundary-layer approaches the scour hole from upstream leading to flow separation at the scour rim that generates vorticity (Melville and Raudkivi 1977; Graf and Istiarto 2002; Dey and Raikar 2007; Kirkil et al. 2008). This region upstream of the horseshoe vortex is described by Melville (1975) as "dead", while Kirkil et al. (2008, (2009) observed a stationary vortex attached to the scour surface next to a secondary horseshoe vortex. Additionally, vortices are randomly shed from the scour rim interacting with the secondary horseshoe vortex (Gobert et al. 2009).

Melville and Raudkivi (1977) identified the streamwise turbulence intensity $\sqrt{\overline{u^{\prime 2}}} / u_{\mathrm{b}}$ to be largest in the intermediate state of the scour development. Whereas Dey and Raikar (2007) document similar distributions of the Reynolds normal stresses $\sqrt{\overline{u^{\prime 2}}} / u_{\mathrm{b}}$ independently of the scour state. According to Kirkil et al. (2009), the shape of the turbulent kinetic energy distribution retains its c-shaped structure when the scour hole develops, as it is the case for a wall-mounted cylinder on a flat bed, e.g., (Paik et al. 2007). Kirkil et al. $(2008,2009)$ observed bimodal velocity distributions around the horseshoe vortex in the scour hole. In contrast, Devenport and Simpson (1990) and Apsilidis et al. (2015) recorded bimodal distributions of the horizontal (wall-parallel) velocity component only inside the wall-parallel jet underneath the horseshoe vortex in front of a cylinder on a flat bed. Dargahi (1990) identified the rotational velocity of the horseshoe vortex, the highmomentum fluid, and the large turbulence levels to be the main impact factors for scouring. For the initial phase of the scour development, Unger and Hager (2007) emphasize the role of the down-flow at the cylinder front being deflected around the cylinder.

The mentioned studies are largely consistent with respect to the time-averaged flow field for a wide range of Reynolds numbers from $\operatorname{Re}=12,700$ to $\operatorname{Re}=398,200$. The similarity of the flow field between the initial and the equilibrium state of the scour development was identified indicating similar processes, as well. Additional effects come into play when the scour hole is present (e.g., vortex shedding from the scour rim) increasing the degree of complexity of this flow configuration. As the previous studies are inconsistent with respect to the turbulence 
levels and structure, and the position and vorticity of the horseshoe vortex, we see the need for a further investigation of the flow field inside a scour hole.

The data which we found in the literature for the maximum out-of-plane vorticity of the horseshoe vortex, for example, vary from $1 u_{\mathrm{b}} / D$ (Das et al. 2013) to values of $10 u_{\mathrm{b}} / D$ (Unger 2006) around the horseshoe vortex centre, while Apsilidis et al. (2015) found approximately $20 u_{\mathrm{b}} / D$ in the flat-bed case. Values for the maximum turbulent kinetic energy around the horseshoe vortex centre obtained from large eddy (LES) and detached eddy simulation (DES) vary from $0.16 u_{\mathrm{b}}^{2}$ at $\operatorname{Re}_{D}=20,000$ (Kirkil et al. 2008), to $0.05 u_{\mathrm{b}}^{2}$ at $\operatorname{Re}_{D}=200,000$ (Kirkil et al. 2009). Measured amplitudes are approximately between $1 u_{\mathrm{b}}^{2}$ (Graf and Istiarto 2002) and $3 u_{\mathrm{b}}^{2}$ (Dey and Raikar 2007), while measured distributions depart from the simulated ones. We attribute the discrepancies in the vorticity and turbulence intensity of the available experimental studies to the low spatial resolution in particular near the scour surface, which was in the range of several millimetres corresponding to more than $0.03 D$. Potential uncertainties of the numerical data caused by modelling effects could not entirely be eliminated due to a lack of reliable highly resolving three-dimensional experimental data, which do not suffer from limitations by simplified or intrusive approaches.

Graf and Istiarto (2002) minimised the impact of the measurement device by mounting a slightly submerged water-filled housing at the water surface containing the ADV profiler. The remaining studies, however, were either intrusive such as hot wire anemometer (Melville and Raudkivi 1977) and submerged ADV (Dey and Raikar 2007), or the experimental set-up was simplified by attaching a half-sided cylinder to the side wall of a transparent flume (Unger and Hager 2007). Therefore, it is unclear how the flow dynamics were affected by the presence of the measurement device itself or by the transparent solid wall in particular in the symmetry plane upstream of the cylinder where the strength of the horseshoe vortex was identified as being the largest.

Non-intrusive experimental data with high spatial resolution are required to be able to link the dynamics of the main vortex systems with predictive morphodynamic scour models. Therefore, to fill in the gap in the scour research, we contribute such data around a circular cylinder at $\mathrm{Re}=20,000$ and 39,000 with a fixed scour geometry at an intermediate stage using a stereoscopic particle image velocimetry (PIV) approach. The measurements were conducted in the symmetry plane upstream of the cylinder as well as in a plane rotated by $45^{\circ}$ with respect to the approach-flow direction at the cylinder's side. Analysing these data, we address the following open questions:

1. Does the turbulence structure of the flow field around a scoured cylinder reveal qualitative and quantitative simi- larities to the one of a wall-mounted cylinder on a flat bed?

2. Can bimodal velocity distributions be found in the scour hole and where do they appear?

3. How does the flow separation at the scour rim occur and does it interfere with the main vortex?

4. How does the near-wall flow and the wall-shear stress develop in the scour?

5. How does the flow structure change with Reynolds number?

In Sect. 2, we describe the details of the experimental set-up and of the measurement technique. The data analysis and discussion follow in Sect. 3. The summary concludes the findings of this study in Sect. 4.

\section{Experimental set-up and data acquisition}

\subsection{Set-up}

The particle image velocimetry (PIV) experiments were conducted in the hydraulic laboratory of the Chair of Hydromechanics at the Technical University of Munich. A cylinder with a diameter of $D=0.1 \mathrm{~m}$ was placed in the symmetry plane of an open water channel with flat bed, which had a length of $20 \mathrm{~m}(200 D)$ and a width of $1.17 \mathrm{~m}(11.7 D)$. The flow depth was $h=0.15 \mathrm{~m}(1.5 D)$.

We have chosen the particular water depth to diameter ratio $h / D=1.5$ because of various reasons. First, it matches previous experiments on scour hole development (Link et al. 2008; Pfleger 2011); second, it provided optical access through the water surface for the stereo-PIV. According to Melville (2008), the scour depth is essentially independent of the water depth if $h / D>1.4$ which is the case in our experiment. Thus, we assume that the water depth has a minor effect on our measured flow fields.

Both of the investigated flow configurations were subcritical, and the corresponding Reynolds numbers were $\mathrm{Re}=20,000$ and 39,000 based on the cylinder diameter $D$ and $u_{\text {sym }}$. The depth-averaged velocity in the symmetry plane of the approach-flow was $u_{\text {sym }}=0.2091 \mathrm{~m} / \mathrm{s}$ and $u_{\text {sym }}=0.3986 \mathrm{~m} / \mathrm{s}$, respectively. Due to the spanwise heterogeneity as a result of the side walls, $u_{\text {sym }}$ is different from the velocity averaged over the cross section of the channel $u_{\mathrm{b}}$ (Schanderl et al. 2017). In our case, the difference was $u_{\mathrm{sym}}=1.026 u_{\mathrm{b}}$.

The approaching flow conditions are of major importance for the flow structure and vortex dynamics in front of a wallmounted cylinder (Schanderl and Manhart 2016). To introduce the initial turbulence at the inlet of the flume, vortex generators were installed as suggested by Counihan (1969). Downstream of these generators, the turbulent channel flow 
could develop naturally for about $130 \mathrm{~h}$ before approaching the measurement section. A detailed documentation of the inflow conditions can be found in Schanderl et al. (2017). The agreement of the mean velocity profile as well as the Reynolds shear and normal stresses with both theoretical and experimental profiles reviewed by Fernholz and Finley (1996) was sufficiently high; thus, we considered the inflow conditions to be both stationary and fully developed.

In contrast to Schanderl et al. (2017), who investigated the flow field in front of a wall-mounted cylinder on a flat plate, we study the flow field inside a scour hole around a circular cylinder at the same and at a lower Reynolds number. The geometry of the scour was taken from Pfleger (2011). The scour evolved under clear-water conditions for $1 \mathrm{~h}$, which corresponded to $14,400 D / u_{\text {sym }}$. The sediment was non-cohesive and had a median diameter of $d_{50}=1.9 \mathrm{~mm}$ or $0.019 D$. This resulted in a scour depth of approximately $0.5 D$, which is smaller than the equilibrium scour depth $(1.2 D)$ and the one used by Kirkil et al. (2008). The scour rim was located at $x / D=-1.4$ in the symmetry plane, the width of the scour hole in spanwise direction was $2.9 D$, and the mean angle of the slope was $\varphi=33.9^{\circ}$. For $\operatorname{Re}=39,000$ and the sediment used, the scour hole can be regarded as in the logarithmic development phase. Pfleger (2011) measured one side of the geometry by a laser distance sensor with a spatial resolution of $5 \mathrm{~mm} \times 5 \mathrm{~mm}$. For modelling the scour geometry as shown in Fig. 1, we assumed symmetry and mirrored the data of Pfleger (2011) as a basis for a fully three-dimensional model. The scour model was milled from aluminium, blackanodized, and coated by a Rhodamine-B-modified $(\mathrm{RhB}) \mathrm{car}$ varnish to reduce light reflections from the surface.

Due to constructional restrictions, the light sheet had to enter the water body from above (Fig. 2a). When the cylinder is placed in the flow, surface waves are induced and refract the incoming light, which undermines the quality of the PIV results. Therefore, we installed a slightly submerged thin slat of acrylic glass at the water surface to suppress bow waves at the cylinder. This element had the size $L \times W \times T=0.15 \mathrm{~m} \times 0.05 \mathrm{~m} \times 0.01 \mathrm{~m}$. The influence of this slat on the approaching flow has been documented by Schanderl et al. (2017). Apart from the region close to the

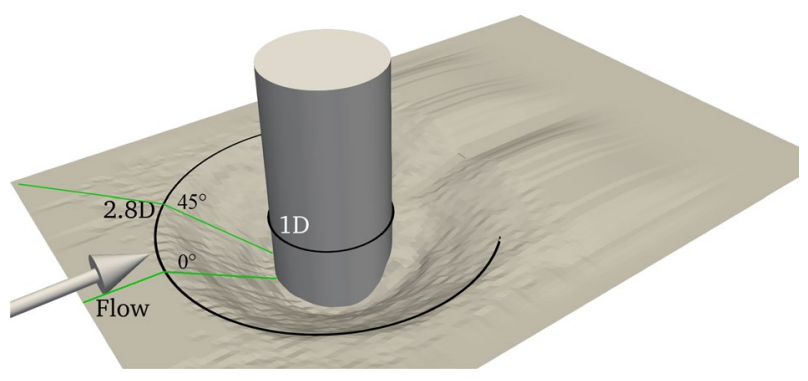

Fig. 1 3D-plot of the scour geometry

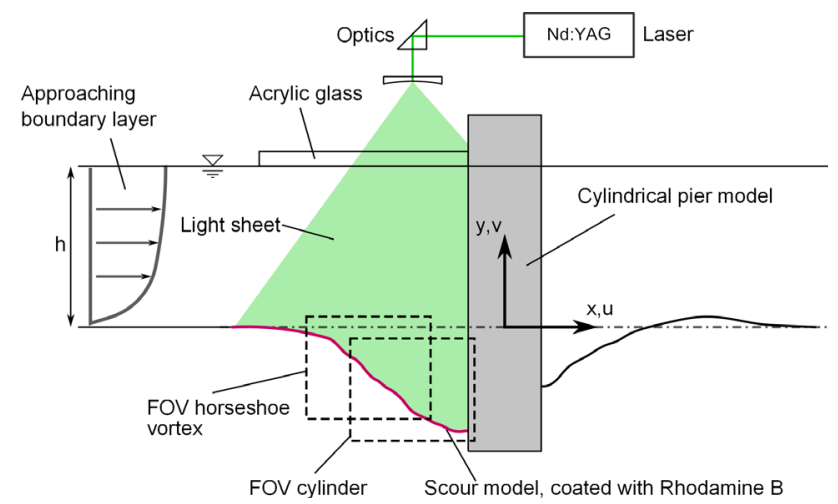

(a) Side view

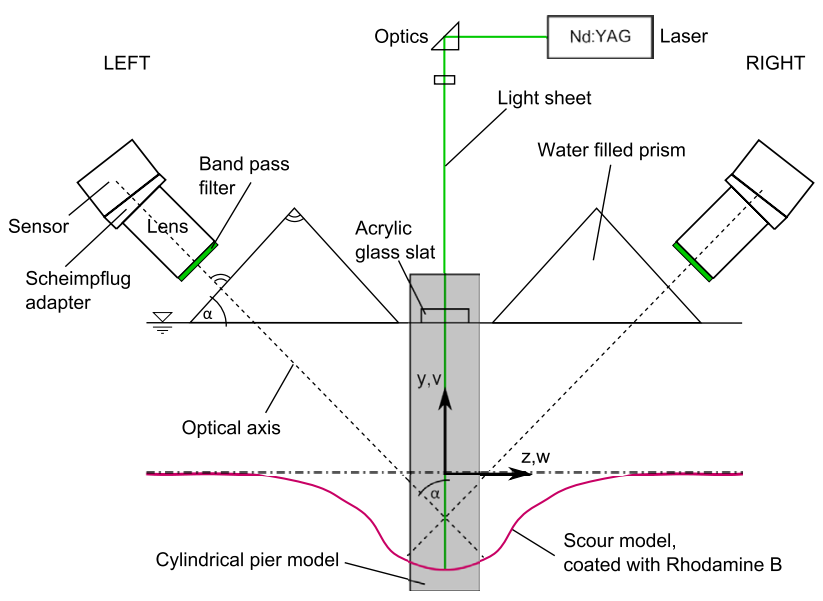

(b) View in flow direction

Fig. 2 Flow configuration and experimental set-up (Jenssen 2019)

water surface, the mean velocity profile as well as the turbulence structure were mainly unaffected by the slat.

Applying stereoscopic PIV (S-PIV) in a water channel entails optic aberrations due to the refraction of light at the water-air interface. Therefore, transparent prisms filled with water were mounted at the water surface to minimise distortions like astigmatism or coma (Adrian and Westerweel 2011). The prisms tilted the refracting interface to be perpendicular with respect to the optical axes of each camera, respectively (Fig. 2b). We followed the angular displacement approach to mount the cameras applying Scheimpflug adapters to enable a homogeneous focussing of the entire image.

The surface of the milled scour model was coated by an RhB-modified car varnish which reduced the reflections from the surface. The incoming laser pulses were of green colour and the reflected light from the scour surface was shifted in wavelength by the fluorescent coating to a reddish colour. By mounting band-pass filters in front of both camera lenses allowed only green light to pass within a band width of $532 \mathrm{~nm} \pm 5 \mathrm{~nm}$ and provided particle images of sufficient quality. The efficiency of the RhB layer was 
not perfect though, and a reflection layer was still visible in the PIV images. The illumination of the tracer particles, however, was homogenized throughout the entire image and not undermined by strong surface reflections.

Furthermore, the reflection layer was also used to detect the position of the scour surface in the PIV images. Sciacchitano and Scarano (2014) suggested to apply a high-pass filter on PIV images to suppress surface reflections. Unfortunately, this filter could not improve the image quality, and therefore, was not applied in our case.

\subsection{Data acquisition}

We used the two dimensional three-component particle image velocimetry (PIV), known as stereoscopic PIV (S-PIV) for collecting the data. Two CCD cameras with a resolution of $2048 \times 2048$ px recorded two velocity components simultaneously via lenses with $105 \mathrm{~mm}$ focal length and an $f$-number of 5.6, which resulted in a magnification factor of about 0.2 . The three components of the velocity field are reconstructed using the information about identical particle displacements in a $2 \mathrm{~mm}$ wide light sheet recorded from two different perspectives. This technique requires accurate calibration to be able to provide resilient data. The mapping function for the stereo-calibration was obtained using a multi-plane target with dots $2 \mathrm{~mm}$ in diameter and a $5 \mathrm{~mm}$ spacing. The target had a relief-like structure with a 1 $\mathrm{mm}$ step in the out-of-plane direction. The three-dimensional shape of the scour geometry did not allow target-traversing.

To meet the requirement of high spatial resolution, two separate measurements were carried out: the first focussing on the cylinder-scour junction and the second focussing on the horseshoe vortex (Fig. 2a). The spatial resolution of the measurements varied between $38.07 \mu \mathrm{m} / \mathrm{px}$ and $36.73 \mu \mathrm{m} / \mathrm{px}$. A joint grid was generated based on the finer resolution, and the time-averaged data were interpolated onto this grid to patch up both overlapping measurements to one data set. As an artefact from this procedure, some results show kinks at the edge of the overlapping region. The plausibility and reliability of the data, however, are not affected by this method.

A $200 \mathrm{~mJ}$ pulsed Nd:YAG laser with a wavelength of 532 $\mathrm{nm}$ was used for the illumination. The data acquisition rate was $f_{\mathrm{s}}=7.25 \mathrm{~Hz}$ and the time delay between each frame was $700 \mu$ s. Due to limitations in computational power, we could record only 1500 images at once, and, in total, we captured 20,000 image pairs for each FOV. This batch processing was leveraged to improve the image quality. While the recorded image pairs were stored, we cleaned the scour model from accumulating particles.

Due to a strong out-of-plane displacement, the loss of particles was high, and the cross-correlation failed eventually to identify the correct in-plane displacements. We did not fill the invalid samples by interpolation from the neighbors, but instead marked them as invalid for the subsequent post-processing in which we treated the valid vectors as statistically independent samples. The number of valid samples $\left(N_{\text {valid }}\right)$ varied across the FOV; therefore, the value in the horseshoe vortex center was taken to be a reasonable representative, since the rate of efficiency of determining valid displacement vectors was the lowest in this sensitive area: The number of valid vectors was $>6263$ for $\mathrm{Re}=39,000$ and $>9838$ for $\operatorname{Re}=20,000$. The standard error in estimating the second and third central moments $\left(\varepsilon\left(M_{n}\right) / \sigma^{n}\right)$ was 0.017 and 0.044 , respectively, for $\mathrm{Re}=39,000$. The trends of the corresponding estimators were statistically converged in this highly turbulent region (Jenssen 2019).

We used $10 \mu \mathrm{m}$ hollow glass spheres to seed the flow. The corresponding relaxation time was $\tau_{\mathrm{P}}=6.11 \times 10^{-6} \mathrm{~s}$ that resulted in a Stokes number of $S t_{\mathrm{k}}=4.85 \times 10^{-3}$; hence, we considered the particles to be able to follow the flow. The influence of the interrogation window size on the resulting vector field is documented by Jenssen (2019) for the flat-bed case. Therefore, we used $16 \times 16 \mathrm{px}$ deformable interrogation windows and an overlap of $50 \%$ to evaluate the instantaneous vector fields. The grid spacing was approximately $2.94 \cdot 10^{-4} \mathrm{~m}$ and the spatial resolution of the interrogation windows was $5.88 \times 10^{-4} \mathrm{~m}$. Together with the $2 \mathrm{~mm}$ light sheet, the measurement volume was $7.16 \times 10^{-4} \mathrm{~cm}^{3}$. This is considerably finer than what was used in the previous studies (Graf and Istiarto 2002; Unger and Hager 2007).

Based on the macroscales, the dissipation rate is estimated a priori for $\mathrm{Re}=39,000$ to $\epsilon_{\text {macro }}=u_{\mathrm{sym}}^{3} / D=0.6333 \mathrm{~m}^{2} / \mathrm{s}^{3}$. Together with the kinematic viscosity of water at $20.2^{\circ} \mathrm{C}\left(1.005 \times 10^{-6} \mathrm{~m}^{2} / \mathrm{s}\right)$, the Kolmogorov length scale is $\eta_{\mathrm{K}}=3.56 \times 10^{-5} \mathrm{~m}$. For $\mathrm{Re}=$ 20,000 the same exercise gives a $\eta_{\mathrm{K}}=5.68 \times 10^{-5} \mathrm{~m}$. The a priori spatial resolution of the S-PIV data is, therefore, estimated to be $16.5 \eta_{\mathrm{K}}$ for $\operatorname{Re}=39,000$ and $10.3 \eta_{\mathrm{K}}$ for $\mathrm{Re}=$ 20,000. In the flat-bed case, this a priori estimation of $\epsilon$ appeared to be conservative, as the highest dissipation rates reached $0.08 u_{\text {sym }}^{3} / D$ near the horseshoe vortex centre (Schanderl et al. 2017). By assuming the dissipation rate to be in the order of magnitude of $0.1 u_{\mathrm{sym}}^{3} / D$, the spatial resolution of the S-PIV data would increase to $9 \eta_{\mathrm{K}}$ for $\mathrm{Re}=$ 39,000

As the spatial resolution is considerably larger than the Kolmogorov scale, we do not expect that variables such as the vorticity variance or the dissipation rate of turbulent kinetic energy can be extracted from our data set. on the other hand, the resolution is fine enough to resolve the timeaveraged flow field and vorticity as well as the Reynolds stresses.

Furthermore, performing PIV measurements close to solid walls entails a potential intersection of the interrogation 
Table 1 Positions of the horseshoe vortex at $0^{\circ}, 45^{\circ}$, and for the flat-bed case

\begin{tabular}{|c|c|c|c|c|c|c|}
\hline \multirow[t]{2}{*}{$\operatorname{Re}$} & \multicolumn{2}{|l|}{$0^{\circ}$} & \multicolumn{2}{|l|}{$45^{\circ}$} & \multicolumn{2}{|l|}{ Flat } \\
\hline & $x / D$ & $x_{n} / D$ & $x / D$ & $x_{n} / D$ & $x / D$ & $x_{n} / D$ \\
\hline 20,000 & -0.89 & 0.053 & -0.91 & 0.09 & -0.68 & 0.051 \\
\hline 39,000 & -0.92 & 0.055 & -0.98 & 0.08 & -0.70 & 0.051 \\
\hline
\end{tabular}

windows by the interface of the wall in the particle images. The affected interrogation window is then subdivided into two areas representing the flow and the solid wall, respectively. The corresponding vector does no longer represent the spatial average of the entire interrogation window but only of the seeded subarea. Since the cameras had to be mounted in an unfavourable set-up in our case (backward scattering and in the main direction of the surface reflections), we decided not to adapt the position of the vectors as suggested by Theunissen et al. (2008) to minimise the near-wall bias. Instead, the minimum distance of the vectors was set to the half of the diagonal length of an interrogation window, i.e., $8 \sqrt{2} \mathrm{px}$, and vectors below this threshold were excluded.

\section{Results}

The results were obtained for $\operatorname{Re}=20,000$ and 39,000. As the Reynolds-number effect on the global flow structures was observed to be small, we mainly focus on the results of $\operatorname{Re}=39,000$ for the sake of comparability to the flat-plate case of Schanderl et al. (2017).

We discuss the time-averaged flow fields in the vertical symmetry plane $\left(0^{\circ}\right)$ upstream of a scoured cylinder as well as at a lateral plane at $45^{\circ}$ with respect to the approach-flow direction. We use coordinate systems aligned with the measurement planes and in which $(x, y, z)$ denote the horizontal, vertical, and azimuthal directions. Note that the azimuthal direction is the out-of-plane direction. Accordingly, $(u, v, w)$ denote the horizontal, vertical, and azimuthal velocity components. The origin of the coordinate system is in the centre of the cylinder at the vertical level of the bottom wall of the approach-flow.

\subsection{Streamlines and magnitude}

The oncoming boundary-layer flow decelerates when approaching the cylinder. This results in a vertical pressure gradient in front of the cylinder which deflects the flow towards the bottom wall and accelerates it along the cylinder surface (Fig. 3a). High-momentum fluid is transported into the scour hole where it impinges on the scour surface. Compared to the flat-bed case, the maximum magnitude of the time-averaged in-plane down-flow increases from $\|\langle\mathbf{u}\rangle\|_{\text {max,flat }}=0.4 u_{\text {sym }}$ up to $\|\langle\mathbf{u}\rangle\|_{\text {max }, \text { scour }} \approx 0.7 u_{\text {sym }}$ (Jenssen

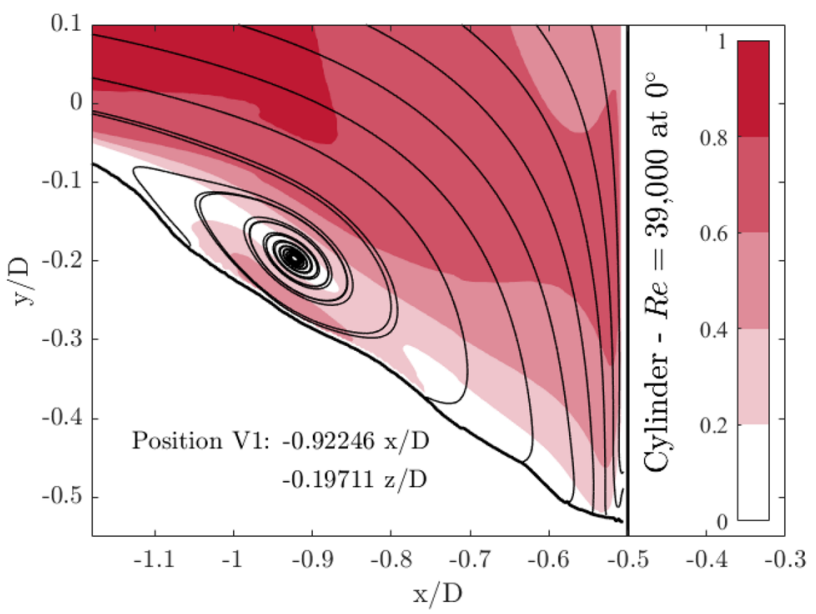

(a) Inplane velocity magnitude $\sqrt{\langle u\rangle^{2}+\langle v\rangle^{2}} / u_{\text {sym }}$

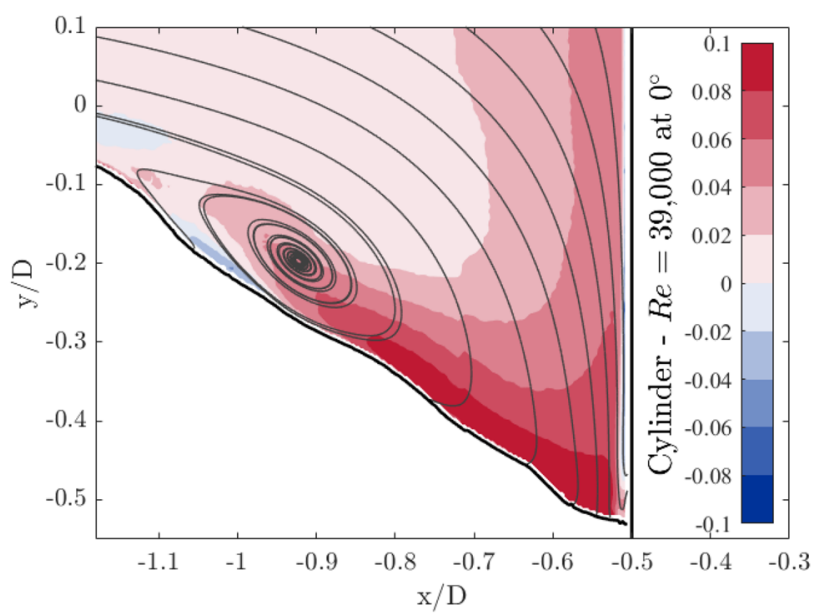

(b) Azimuthal component $\langle w\rangle / u_{\text {sym }}$

Fig. 3 Time-averaged streamlines of the flow in the symmetry plane $\left(0^{\circ}\right)$ upstream of a scoured cylinder flooded with a the magnitude of the in-plane velocity field and $\mathbf{b}$ the azimuthal component. Normalized by $u_{\text {sym }}$

2019). The corresponding maximum values of the downflow-magnitude observed by Melville and Raudkivi (1977), Graf and Istiarto (2002), and Dey and Raikar (2007) are very $\operatorname{similar}\left(\approx 0.6 u_{\text {sym }}\right)$.

Like in the flat-bed case (Fig. 4), the stagnation point S3 appears near the foot of the cylinder at the scour surface and the flow is deflected in all directions: towards the cylinder, in the uphill direction, and in the spanwise direction circumferentially around the cylinder. In Fig. 3b, the azimuthal 


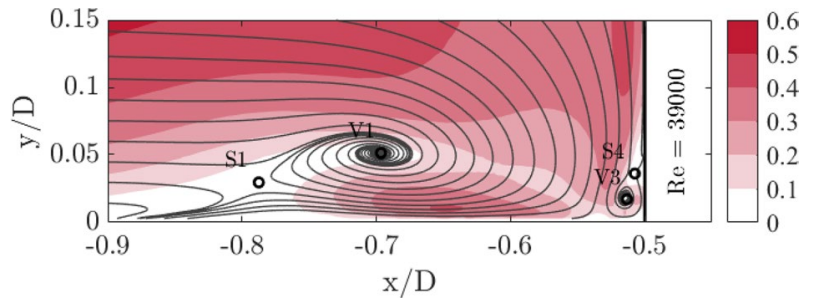

Fig. 4 Time-averaged in-plane velocity magnitude on flat bed $\sqrt{\langle u\rangle^{2}+\langle v\rangle^{2}} / u_{\text {sym }}($ Jenssen 2019)

velocity component $\langle w\rangle / u_{\text {sym }}$ is shown. When approaching the cylinder, the values are close to zero due to symmetry. At S3, however, the flow becomes three-dimensional indicated by an increasing azimuthal velocity. Graf and Istiarto (2002), for example, documented similar out-of-plane velocity values $\left(\approx 0.06 u_{\text {sym }}\right)$ at the cylinder front. Our expectation is that the magnitude of the azimuthal velocity would decay with a larger number samples because of symmetry.

A small foot vortex appeared in the flat-bed case caused by the deflection of the down-flow at a distance of $0.03 \mathrm{D}$ from the front of the cylinder (V3 in Fig. 4). A similar junction vortex has been observed by Kirkil et al. (2008) and Kirkil and Constantinescu (2010) in DES of the flow in an equilibrium scour hole at two different Reynolds numbers. We cannot find such a vortex in our data when plotting streamlines. The streamwise and vertical velocity components, however, change their signs near the cylinder foot indicating that there might exist a small foot vortex, too, in the scour hole. However, this vortex-if present-would be much smaller than the one over a flat plate. Note that our interrogation windows are marginally smaller than half of the distance of foot vortex to the cylinder in the flat-bed case.

The flow accelerates along the wall uphill of the stagnation point $\mathrm{S} 3$ until a wall-parallel jet is formed. As in the flat-bed case, parts of the down-flow roll up and form the horseshoe vortex (V1) bending around the cylinder. The time-averaged centres of V1 are given in Table 1. They were detected by the point of intersection of the zero isolines of the horizontal and the vertical velocity components. The vortex centre is located more upstream than in the flat-bed case. The distance between vortex and wall denoted by $x_{n}$ is only slightly larger than in the flat-bed case. Note that the difference between the flat bed and the scour would be only $0.1 \mathrm{~mm}$ and $0.4 \mathrm{~mm}$ for $\mathrm{Re}=20,000$ and 39,000, respectively, which is probably below the accuracy of determining the position of the wall.

Between the vortex centre and the scour surface, the wall jet intensifies strongly. The amplitude of the velocity magnitude is $\|\langle\mathbf{u}\rangle\|_{\max }>0.5 u_{\text {sym }}$, which is considerably larger than the corresponding flat-bed velocity of $0.3 u_{\text {sym }}$ (Jenssen 2019). The maxima of the down-flow and the wall jet velocities indicate that the intensity of the horseshoe vortex is amplified in the scour hole with respect to the flat-bed case.

The wall jet slows down after having passed the horseshoe vortex centre. Further uphill, in the region between $\mathrm{V} 1$ and the scour rim, the velocity magnitude becomes very small. Kirkil et al. (2009) observed a saddle point and a secondary vortex here. We cannot confirm these observations. Our time-averaged data suggest instead that there is a large recirculation zone with very small back-flow velocities. The interface between this recirculation and the flow above forms a shear layer indicating a flow detachment from the scour rim, which was also observed by Dey and Raikar (2007), for example.

At the side of the cylinder at $45^{\circ}$ with respect to the approach-flow, the in-plane down-flow along the cylinder as well as the uphill directed wall jet and the horseshoe vortex are still apparent (Fig. 5a, b). Above the horseshoe vortex, the intensity of the in-plane velocity magnitude decreases, whereas the one of the down-flow is still approximately $0.5 u_{\text {sym }}$ and the maximum in-plane wall-jet velocity underneath the horseshoe vortex is about $0.6 u_{\text {sym }}$. The azimuthal velocity component is predominant at $45^{\circ}$. In the down-flow along the cylinder, the out-of-plane velocity component exceeds $1.0 u_{\text {sym }}$, and even in the near-wall jet downhill of the horseshoe vortex at $x / D \approx-0.8$, the azimuthal component is about $0.5 u_{\text {sym }}$. Another difference with respect to the symmetry plane is the shifted time-averaged position of the horseshoe vortex: further uphill and lifted from the scour surface (see Table 1). At the cylinder foot, the scour surface flattened; thus, the surface reflections increased that is leading to a lack of data in this region.

\subsection{Vorticity}

We discuss the time-averaged out-of-plane vorticity $\omega_{z}=\left(\frac{\partial\langle\nu\rangle}{\partial x}-\frac{\partial\langle u\rangle}{\partial y}\right) \cdot \frac{D}{u_{\mathrm{sym}}}$ in the symmetry plane and at $45^{\circ}$ (Fig. 6a, b). In case of the azimuthal plane at $45^{\circ}$, the vorticity is computed using the local in-plane velocity gradients.

In the symmetry plane, the vorticity is largest in the horseshoe vortex centre: $\omega_{z}=20 u_{\text {sym }} / D$ at $\operatorname{Re}=20,000$ and $\omega_{z}=17 u_{\text {sym }} / D$ at $\operatorname{Re}=39,000$. These values are larger than in the flat-bed case (Jenssen 2019), which are below $17 u_{\text {sym }} / D$ for both Reynolds numbers. This indicates that the increasing maximum velocity value in the scour hole can be partly explained by a slight increase of the vorticity in the vortex.

In the flat-bed case, fluid from $y=0.05 D$ to $y=0.15 D$ is entrained into the vortex. Whereas in the scour, all fluid from below $y=0.1 D$ is entrained. This means that the original vorticity on a streamline entering the vortex is larger in the 


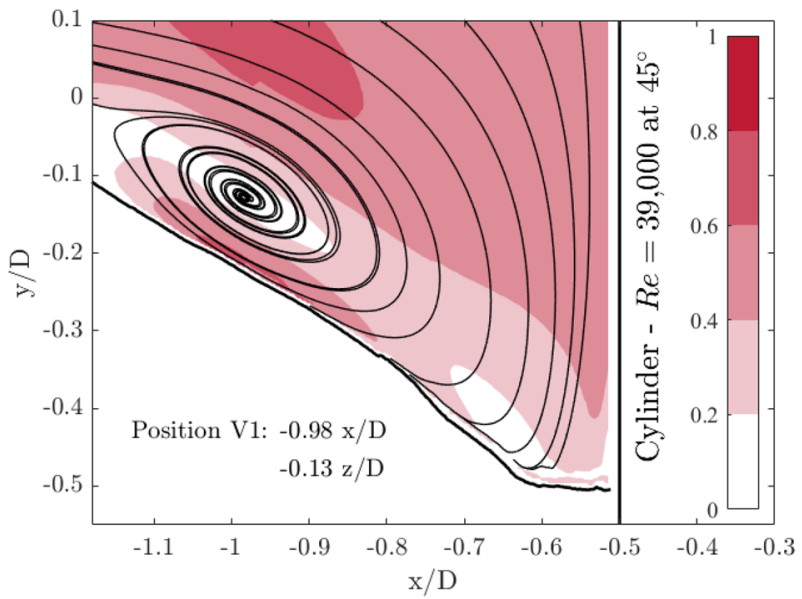

(a) Inplane velocity magnitude $\sqrt{\langle u\rangle^{2}+\langle v\rangle^{2}} / u_{\text {sym }}$

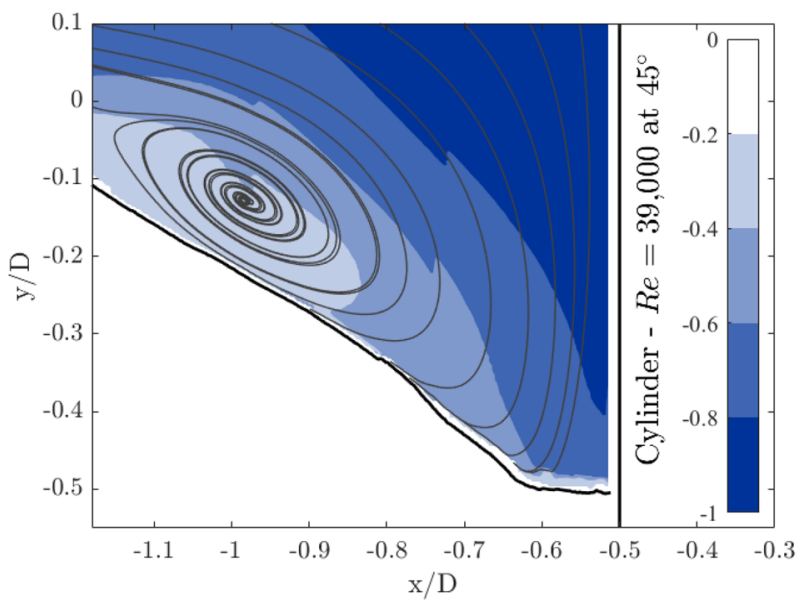

(b) Azimuthal component $\langle w\rangle / u_{\text {sym }}$

Fig. 5 Time-averaged streamlines of the flow in a vertical plane at $45^{\circ}$ at the side of a scoured cylinder flooded with a the magnitude of the in-plane velocity field and $\mathbf{b}$ the azimuthal component. Normalized by $u_{\text {sym }}$

scoured-cylinder case which could be a factor explaining the larger vorticity in the scour hole.

Our vorticity magnitudes are larger than what was reported by other authors. Graf and Istiarto (2002) observed only "weak" vorticity of about $7 u_{\mathrm{b}} / D$, Dey and Raikar (2007) found values around $3 u_{\mathrm{b}} / D$, and Das et al. (2013) reported values around $1 u_{\mathrm{b}} / D$. In the PIV measurements of a half-cylinder configuration, Unger (2006) found values below $10 u_{\mathrm{b}} / D$ in the various investigated cases with the majority lying below $5 u_{\mathrm{b}} / D$.

We observe layers of large counter-clockwise vorticity along the cylinder front and along the scour surface, which are generated by the boundary layers of the down-flow at the cylinder front and by the near-wall jet inside the scour hole, respectively. Both layers grow in the local streamwise direction. The thickness of the vorticity layer under the wall

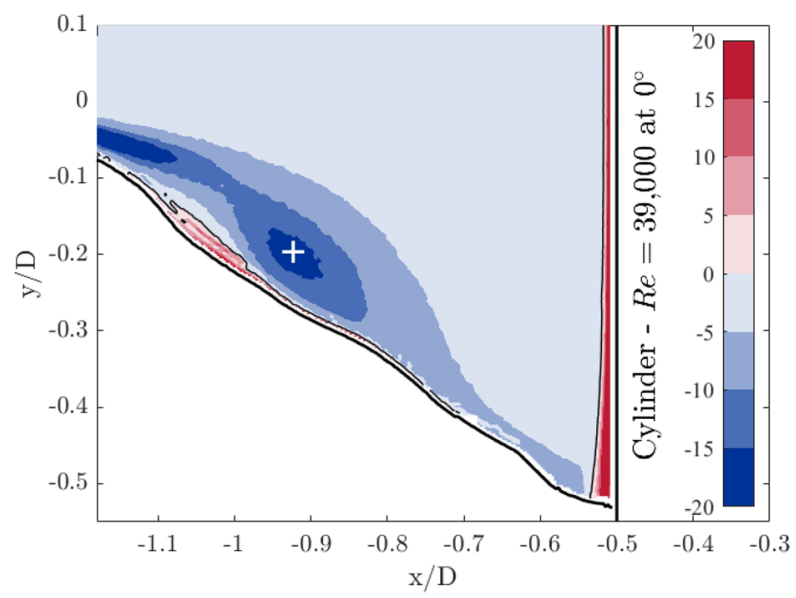

(a) $0^{\circ}$

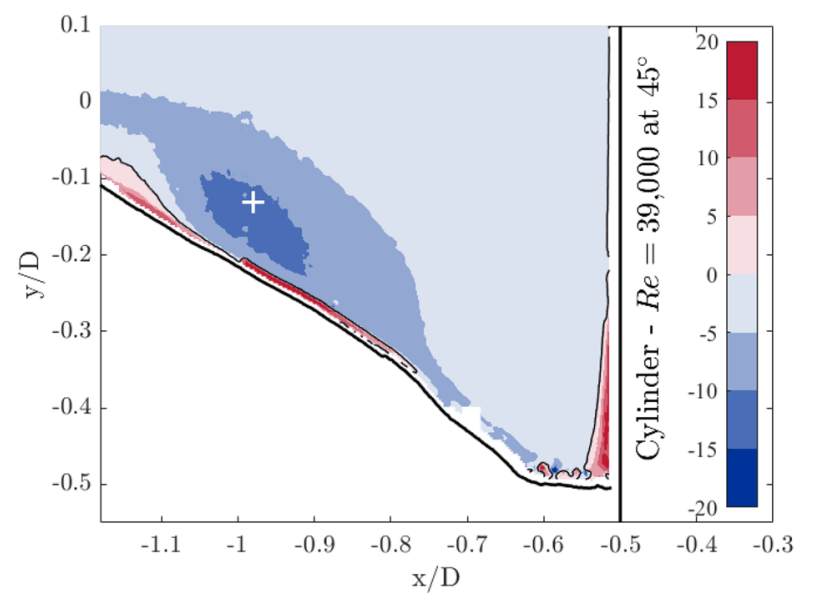

(b) $45^{\circ}$

Fig. 6 Time-averaged vorticity $\omega_{z}=\left(\frac{\partial\langle v\rangle}{\partial x}-\frac{\partial\langle u\rangle}{\partial y}\right) \frac{D}{u_{\mathrm{sym}}}$ in the symmetry plane a upstream and in a vertical azimuthal plane at $45^{\circ} \mathbf{b}$ of a scoured cylinder. Cross indicates V1 and the solid black line indicates the isoline $\omega_{z}=0$

jet grows strongly uphill of the horseshoe vortex, which is similar to the flat-bed case (Jenssen 2019).

The approaching boundary layer detaches at the scour rim at $y / D=0$ and $x / D=-1.4$. The detached shear layerindicated by negative (i.e., clockwise) vorticity-enters the measurement area around $y / D=-0.05$. Following the streamlines, the shear layer seems to merge with the horseshoe vortex as both rotate in the clockwise direction and reveal a similar intensity of $\omega_{z}$. From the point of detachment on, the intensity of the vorticity decreases along the streamlines before it increases again at the centre of the horseshoe vortex. In the detaching shear layer uphill of the horseshoe vortex, we assume the formation of Kelvin-Helmholtz $(\mathrm{KH})$ vortices.

While the horseshoe vortex bends around the cylinder, the distribution of the out-of-plane vorticity remains similar 
to the one in the symmetry plane. Although the intensity of the vorticity at V1 decreases, it reaches values of more than $10 u_{\text {sym }} / D$ (Fig. 6b). The uphill directed jet is still present as the positive vorticity layer indicates an increasing thickness uphill of the horseshoe vortex. The down-flow, however, is deflected in the azimuthal direction out of the light sheet plane, and therefore, the resulting developing boundary layer along the cylinder surface is much weaker and the positive vorticity layer is less pronounced as in the symmetry plane.

\subsection{Turbulent kinetic energy}

Figure 7 presents the distribution of the turbulent kinetic energy (TKE) $k=0.5\left\langle u_{i}^{\prime 2}\right\rangle / u_{\mathrm{sym}}^{2}$. The points P1-P5 are discussed in Sect. 3.7. The maximum of the TKE can be found around the centre of the horseshoe vortex. It is connected to

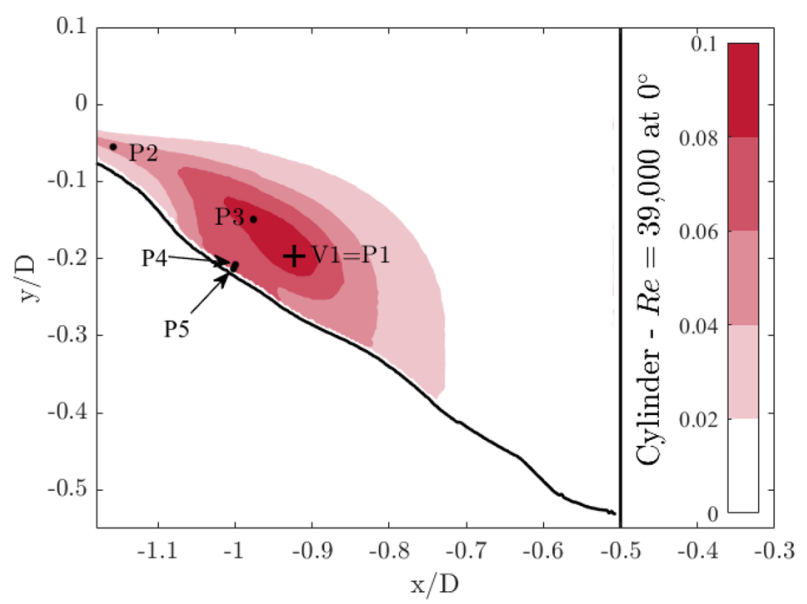

(a) $0^{\circ}$

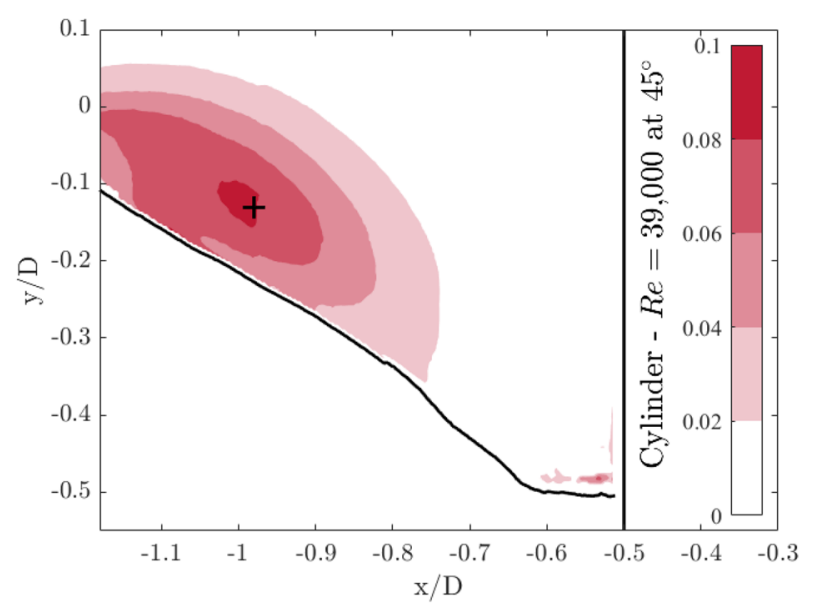

(b) $45^{\circ}$

Fig. 7 Time-averaged turbulent kinetic energy $k / u_{\text {sym }}^{2}$ in the symmetry plane a upstream and in a vertical azimuthal plane at $45^{\circ}$ of a scoured cylinder (b). Cross indicates V1 a thin layer of enhanced TKE in the shear layer detaching from the rim of the scour hole. A near-wall peak can be identified at the scour surface under the horseshoe vortex. This might be linked to the deceleration of the near-wall jet. There is no enhanced TKE at the foot of the cylinder that could indicate a foot vortex as in the flat-bed case (Schanderl et al. 2017) and as it was observed in a scour hole by Kirkil et al. (2008) and Kirkil et al. (2009) performing numerical simulations.

The TKE distribution bears similarities to the c-shaped structure found in the flat-bed case by Devenport and Simpson (1990); Paik et al. (2007); Apsilidis et al. (2015); Schanderl et al. (2017). This is an indicator that the dynamics of the vortex system in the scour hole is similar to that on a flat plate. However, the amplitude of $k$ is approximately by 10-20\% larger than in the flat-bed case (Jenssen 2019). Furthermore, the amplitude of $k$ at the horseshoe vortex centre in the symmetry plane decreases slightly with increasing Reynolds number as $k_{20,000}=0.103$ and $k_{39,000}=0.093$.

The trace of TKE from the scour rim to the centre of the horseshoe vortex suggests that Kelvin-Helmholtz vortices are generated in the detached shear layer, and grow when being transported towards the horseshoe vortex. This is in line with vortices observed and discussed in numerical simulations by Gobert et al. (2009) and Kirkil et al. (2010). Our measured TKE distribution shows some differences when compared to the ones from LES and DES by Kirkil et al. (2008) and Kirkil et al. (2009). While they found a c-shaped distribution as well, their amplitudes are considerably larger in the horseshoe vortex centre. Additionally, the trace of enhanced TKE from the shear layer separating from the scour rim was not that intense in the numerical results.

The c-shape of the TKE distribution is preserved when the horseshoe vortex bends around the cylinder, whereas the amplitude of $k$ decreases (Fig. 7b). The TKE decay is stronger at $\operatorname{Re}=20,000: k_{20,000,45^{\circ}}=0.074$ and $k_{39,000,45^{\circ}}=0.084$. The TKE peak is shifted in the uphill direction according to the time-averaged location of the horseshoe vortex centre and the distribution has a larger spatial extent than in the symmetry plane. The attenuation of the TKE in the vortex is consistent with the smaller vorticity at $45^{\circ}$ which indicates smaller velocity gradients. Spatial oscillations of the horseshoe vortex centre would, consequently lead to smaller velocity fluctuations.

Although the shape of the TKE distribution in the scour is similar to the one in the flat-bed case, the individual distributions of the in-plane Reynolds normal stresses differ from the results of Schanderl et al. (2017). The distribution of $\left\langle u^{\prime} u^{\prime}\right\rangle$ (Fig. 8a) is separated in three regions: (i) a peak around V1; (ii) a near-wall peak; and (iii) a peak in the shear layer detaching from the scour rim. The normal stresses in the vertical direction $\left\langle v^{\prime} v^{\prime}\right\rangle$ (Fig. 8c) reveal a large peak uphill of the time-averaged position of the horseshoe vortex. 


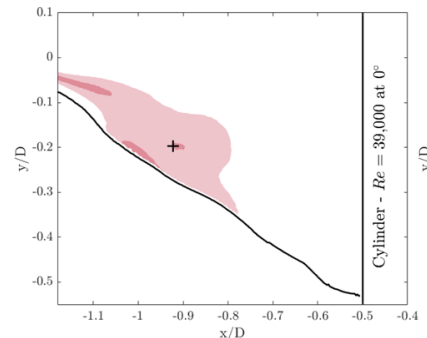

(a) $\left\langle u^{\prime} u^{\prime}\right\rangle / u_{\mathrm{sym}}^{2}$

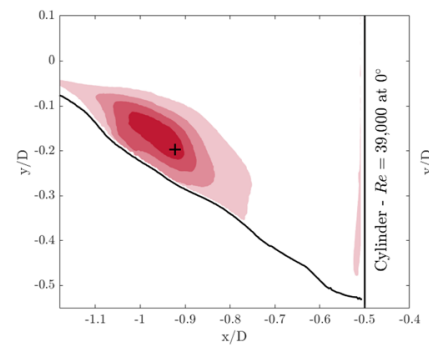

(c) $\left\langle v^{\prime} v^{\prime}\right\rangle / u_{\text {sym }}^{2}$

0 0.02 0.04 0.06 0.08 0.1

Fig. 8 Time-averaged Reynolds normal stresses in the symmetry plane in front of a scoured cylinder at $\mathrm{Re}=39,000$ : left column in the Cartesian coordinate system $(\mathbf{a}, \mathbf{b})$; right column in the scour-oriented system $(\mathbf{c}, \mathbf{d})$. Cross indicates V1

In the flat-bed case, the vertical fluctuations and the resulting normal stresses were caused by horizontal oscillations of the vortex system, and, consequently, reveal an elliptical shape. The lower branch of the c-shape in the TKE distribution is assigned to be the footprint of the wall-parallel jet, and thus, caused by fluctuations in the direction of the jet. As the jet is horizontally aligned in the flat-bed case, the velocity fluctuations in the jet coincide with those of the horizontal velocity component. This correlation is apparently not valid any more in the scour, as the distributions of the vertical and the horizontal Reynolds normal stresses differ from those of the flat-bed case. When summing them up, however, the distribution of the resulting TKE in the scour configuration matches the one of the flat-bed case. Therefore, we suppose that the vortex oscillations occur parallel to the bounding wall, i.e., either in the horizontal direction (flat-bed case) or parallel to the inclination of the scour surface. Dey and Raikar (2007), too, documented that the main axis of the elliptical horseshoe vortex is oriented parallel to the scour surface. To confirm and extend this observation, we analysed the Reynolds normal stresses in a rotated coordinate system according to the local inclination of the scour surface.

Therefore, the Cartesian coordinates $\mathbf{x}=(x, y, z)^{\mathrm{T}}$ were transformed into a scour-oriented system $\hat{\mathbf{x}}=\left(x_{r}, x_{n}, x_{a}\right)^{\mathrm{T}}$. The subscripts stand for radial, normal, and azimuthal, respectively. The $x_{r}$-component points towards the cylinder axis and is aligned parallel to the scour surface. The $x_{n}$ -component is the component normal to the scour surface, and the $x_{a}$-component represents the azimuthal component with respect to the cylinder and points out of the light sheet plane.

The Reynolds normal stresses in the scour-oriented coordinate system change with respect to the Cartesian orientation and are shown in the right column of Fig. 8. The similarity between the Reynolds stress distributions in a scour hole with those on a flat bed becomes apparent. Like in the flat-bed case, the wall-normal fluctuations $\left\langle u_{n}^{\prime} u_{n}^{\prime}\right\rangle$ are elliptically distributed around the vortex core (Fig. 8d) and can mainly be explained by wall-parallel shifts of the horseshoe vortex. The fluctuations in the wall-parallel velocity component $\left\langle u_{r}^{\prime} u_{r}^{\prime}\right\rangle$, however, are strong in the near-wall jet uphill of the horseshoe vortex centre similar to the flat-bed case (Schanderl et al. 2017). Furthermore, Schanderl et al. (2017) attributed the turbulence structure in this region to the wallnormal flapping of the near-wall jet. This agrees with the concept of zero- and back-flow modes proposed by Devenport and Simpson (1990) for the flat-bed case, which was also observed in DES by Kirkil et al. (2009) for the scour.

The approaching flow separates at the scour rim inducing a shear layer and vortex shedding. According to the amplified $\left\langle u_{r}^{\prime} u_{r}^{\prime}\right\rangle$ stresses and the small wall-normal stresses $\left\langle u_{n}^{\prime} u_{n}^{\prime}\right\rangle$ along the shear layer upstream of V1 (Fig. 8b,d), additional turbulent kinetic energy is fed into the horseshoe vortex by the shedding vortices from the scour rim. This contribution of $\left\langle u_{r}^{\prime} u_{r}^{\prime}\right\rangle$ is significantly large up to $0.07 u_{\text {sym }}^{2}$ as the corresponding value at V1 is $0.057 u_{\text {sym }}^{2}$.

To sum up, (i) the vortex oscillations are oriented parallel to the surface of the scour; (ii) a near-wall jet structure develops like in the flat-bed case; and (iii) the shear layer separating from the scour rim introduces additional turbulent kinetic energy. The detailed documentation of the Reynolds stresses could not be found in the literature so far. In fact, Dey and Raikar (2007) reported that the distributions of $\left\langle u^{\prime} u^{\prime}\right\rangle,\left\langle v^{\prime} v^{\prime}\right\rangle$, and $\left\langle w^{\prime} w^{\prime}\right\rangle$ are similar to each other, which is apparently an artefact of an insufficient spatial resolution.

In Fig. 9a, b, the shear stresses with respect to the original (Cartesian) and the scour-oriented coordinate system $\left\langle u^{\prime} v^{\prime}\right\rangle$ and $\left\langle u_{r}^{\prime} u_{n}^{\prime}\right\rangle$ are shown, respectively. Due to the coordinate transformation, the structure of the Reynolds shear stress changed completely. In the Cartesian system (a), the entering shear layer and the wall-parallel uphill directed fluctuations in the wall jet are predominant, while the horseshoe vortex centre does not indicate a local amplitude. When rotating the coordinate system parallel to the scour surface, a peak of negative shear stress arises uphill of V1, while the shear layer from the scour rim is not visible any more. In general, the amplitudes of the shear stresses in both systems are a little lower than the corresponding normal stresses (Fig. 8). 


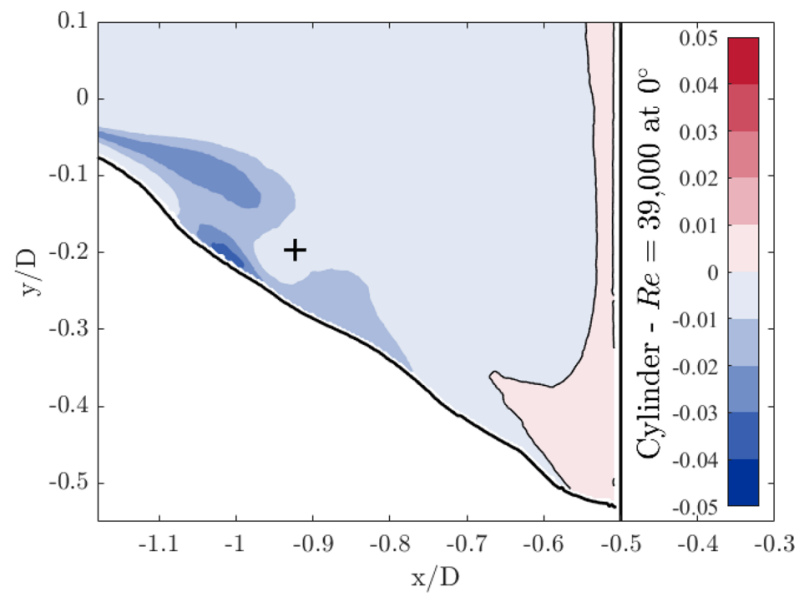

(a) $\left\langle u^{\prime} v^{\prime}\right\rangle / u_{\mathrm{sym}}^{2}$

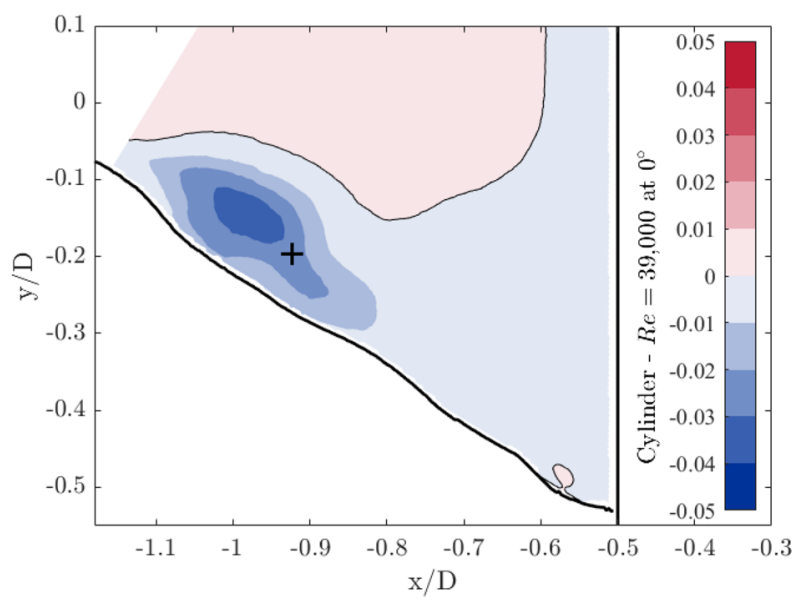

(b) $\left\langle u_{r}^{\prime} u_{n}^{\prime}\right\rangle / u_{\text {sym }}^{2}$

Fig. 9 Time-averaged Reynolds shear stresses in the symmetry plane in front of a scoured cylinder at $\operatorname{Re}=39,000$ normalized by $u_{\text {sym }}^{2}$; in the Cartesian coordinate system $\left\langle u^{\prime} v^{\prime}\right\rangle(\mathbf{a})$; and in the scour-oriented system $\left\langle u_{r}^{\prime} u_{n}^{\prime}\right\rangle$ (b). Cross indicates V1 and the solid black line the zero isoline

In the scour-oriented system, we do not observe the enhanced levels of shear stresses near the wall which would indicate a turbulent momentum transport towards the scour surface. By analysing both, the Reynolds shear and normal stresses, we see that the main direction of the stresses changes when the flow enters the scour. Following the streamlines, the main stresses are subjected to a change of the direction: (i) upstream of the scour hole, the term $\left\langle u^{\prime} u^{\prime}\right\rangle$ is dominant; (ii) when entering the scour hole, the stresses occur mainly in the scour-parallel direction $\left\langle u_{r}^{\prime} u_{r}^{\prime}\right\rangle$; and (iii) around the time-averaged location of the horseshoe vortex, the vertical stresses $\left\langle v^{\prime} v^{\prime}\right\rangle$ are prevailing. Note that the wall-normal stresses $\left\langle u_{n}^{\prime} u_{n}^{\prime}\right\rangle$ become more important at V1, but do not exceed the vertical ones.

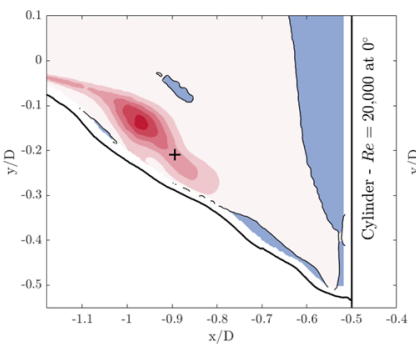

(a) $R e=20,000$ at $0^{\circ}$

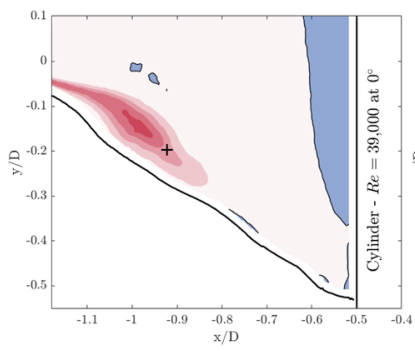

(c) $R e=39,000$ at $0^{\circ}$

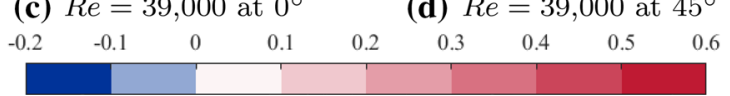

(b) $R e=20,000$ at $45^{\circ}$

(d) $R e=39,000$ at $45^{\circ}$
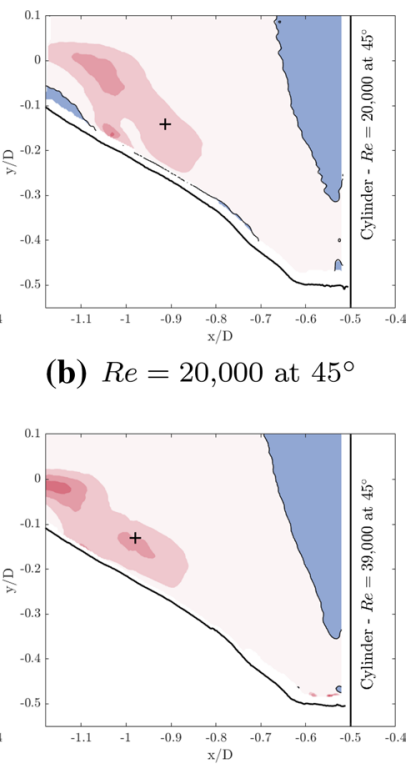
6

Fig. 10 Time-averaged total production $P D / u_{\text {sym }}^{3}$ of turbulent kinetic energy upstream of a scoured cylinder: left column symmetry plane $\left(0^{\circ}\right)$; right column vertical azimuthal plane at $45^{\circ}$ for $\operatorname{Re}=20,000(\mathbf{a}$, c) and $\operatorname{Re}=39,000(\mathbf{b}, \mathbf{d})$. Cross indicates V1 and the solid black line indicates the zero isoline

\subsection{Production of turbulent kinetic energy}

In a next step, we study the production of the turbulent kinetic energy, which is computed as:

$P=-\left\langle u_{i}^{\prime} u_{j}^{\prime}\right\rangle \frac{\partial\left\langle u_{i}\right\rangle}{\partial x_{j}}$

The total production is a scalar field as a result from the sum of the nine terms in Eq. (1). The S-PIV approach provides all three components of the velocity vector field. However, only the in-plane gradients such as $\partial / \partial x$ and $\partial / \partial y$ can be directly calculated, and by assuming incompressibility, the gradient $\partial w / \partial z$ can be obtained from the divergence of the vector field. As a consequence, the gradients $\partial u / \partial z$ and $\partial v / \partial z$ are missing in the calculation of the production term. In the symmetry plane $\left(0^{\circ}\right)$, we assume these gradients to be approximately zero, whereas they are non-zero at $45^{\circ}$ and provide an unidentified contribution to the total production term. Figure 10 shows the normalized production of TKE $\left(P D / u_{\text {sym }}^{3}\right)$ for both $\operatorname{Re}=20,000$ and $\operatorname{Re}=39,000$.

The spatial distribution of the TKE production is characterised by the shear layer detaching from the scour rim and by the horseshoe vortex. The maximum value of $P$ can be found in a region upstream of V1 in which the KH vortices interfere with the horseshoe vortex. The amplitude of $P$ at 
$0^{\circ}$ decreases with increasing Reynolds number from $P_{20,000}=0.54 u_{\text {sym }}^{3} / D$ to $P_{39,000}=0.43 u_{\text {sym }}^{3} / D$. At $45^{\circ}$, the $P$-amplitude decreases in general and between the Reynolds numbers. The first result indicates that the horseshoe vortex draws its TKE from the $0^{\circ}$-plane. Due to the sideways deflection of the approaching flow, the interference of the $\mathrm{KH}$ vortices and the horseshoe vortex decreases. Furthermore, the spatial distribution of the production at $\mathrm{Re}=$ 20,000 reveals a lower branch from the region of its largest amplitude towards the scour surface, which coincides with the location of the lower branch of the c-shaped TKE distribution. In the flat-bed case, there is a local maximum of TKE production, which is probably not resolved in our measurements due to wall reflections.

In front of the cylinder, the vertical downward acceleration leads to a transformation of TKE into mean kinetic energy as the negative values of $P$ indicate. The same applies for the region along the scour surface (in particular for Re $=20,000)$. The flow accelerates from the stagnation point S3 onwards in the uphill direction, and therefore, TKE is transferred into mean kinetic energy. Further uphill, when the slope reduces around $x / D=-0.8$, the flow decelerates, and consequently, the production changes sign and becomes positive underneath the horseshoe vortex. This process is similar to what has been described in the flat-bed case by Schanderl et al. (2017).

Figure 11 shows the production of TKE split into the sum of normal stress production (a), i.e., $i=j$ in Eq. (1), and sum of shear stress production (b), i.e., $i \neq j$ in Eq. (1) in the Cartesian coordinate system. Enhanced levels of shear stress production mark the shear layer detaching from the scour rim, whereas it is around zero in the rest of the measurement area, in particular at the horseshoe vortex. The production by normal stresses reveals a peak upstream of V1 at the same location where $\left\langle v^{\prime} v^{\prime}\right\rangle$ has its largest amplitude. Therefore, we conclude that the production of TKE upstream of V1 is mainly governed by vertical stresses. Note that distinguishing into 'normal' and 'shear' stress is highly dependent on the coordinate system, and the situation is inverted in the scour-oriented reference frame. The 'normal' stress production mainly generated by $\left\langle u_{r}^{\prime} u_{r}^{\prime}\right\rangle$ has a similar distribution as the 'shear' stress production in Fig. 11b, while the 'shear' stress production in the scour-oriented frame is governed by $\left\langle u_{r}^{\prime} u_{n}^{\prime}\right\rangle$, which is caused by the vertical stresses $\left\langle v^{\prime} v^{\prime}\right\rangle$ contributing to Fig. 11a (Jenssen 2019).

\subsection{Velocity profiles}

To gain a deeper understanding of the near-wall jet, we analyse the time-averaged wall-parallel velocity component and the in-plane Reynolds shear stress in detail. Doing so, we select three streamwise positions at which we compare our

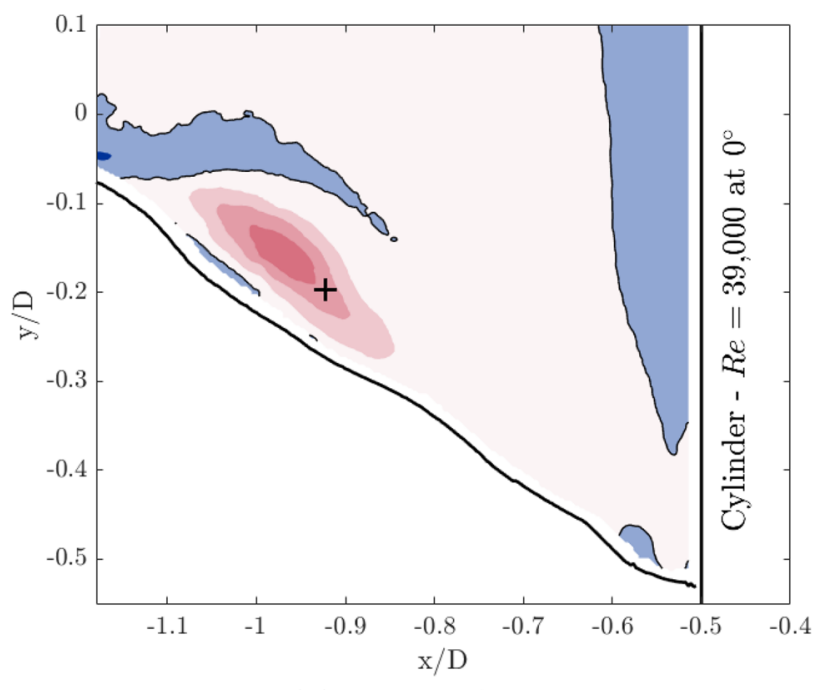

(a) $P_{i, i} D / u_{\mathrm{sym}}^{3}$

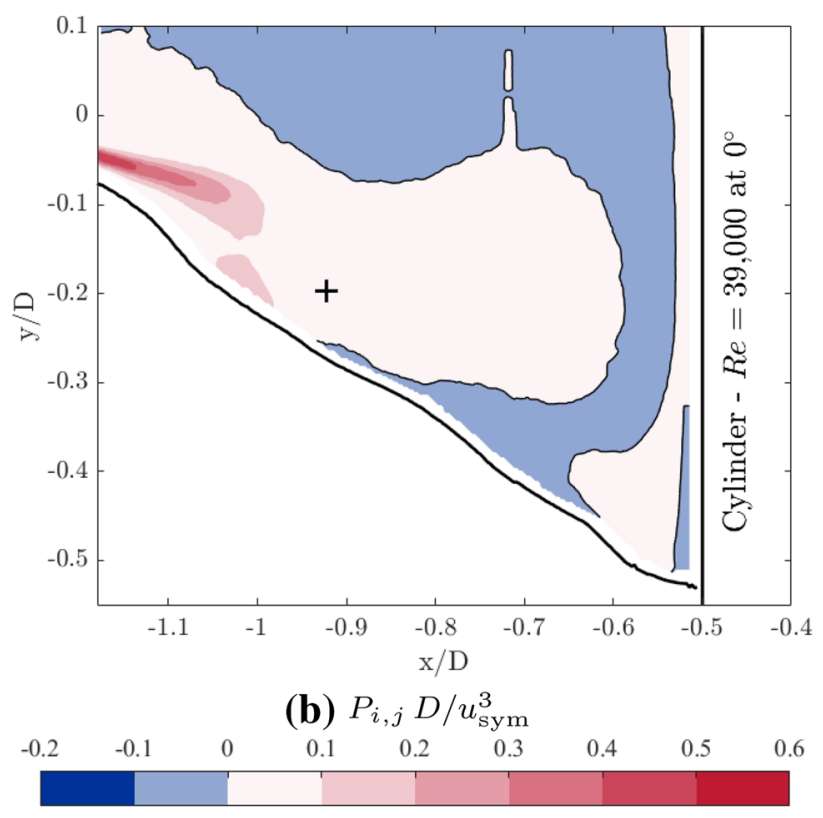

Fig. 11 Time-averaged production of turbulent kinetic energy upstream of a scoured cylinder at $\operatorname{Re}=39,000$ is split into the sum of normal production, i.e., $i=j(\mathbf{a})$, and into the sum of shear production, i.e., $i \neq j$ (b). Cross indicates V1 and the solid black line indicates the zero isoline

wall-normal profiles with those measured above a flat plate (Fig. 12): at the foot of the c-shaped TKE $(x / D \approx-1.0)$, through the horseshoe vortex centre $\mathrm{V} 1$, and between the cylinder and $\mathrm{V} 1(x / D \approx-0.81)$.

The wall-normal coordinate is denoted as $x_{n}$ and the wallparallel velocity as $u_{r}$, which corresponds to the $y$-coordinate and the $u$-velocity in the flat-bed case, respectively. The profiles of the flat-bed case were extracted accordingly, but the horizontal positions varied as the horseshoe vortex position varied, as well. To take this difference into account, 


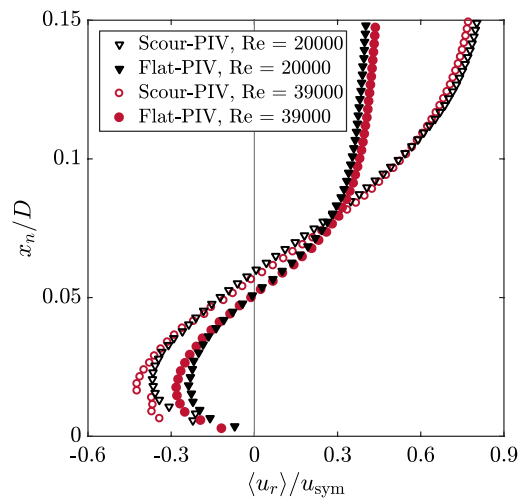

(a) At TKE-foot

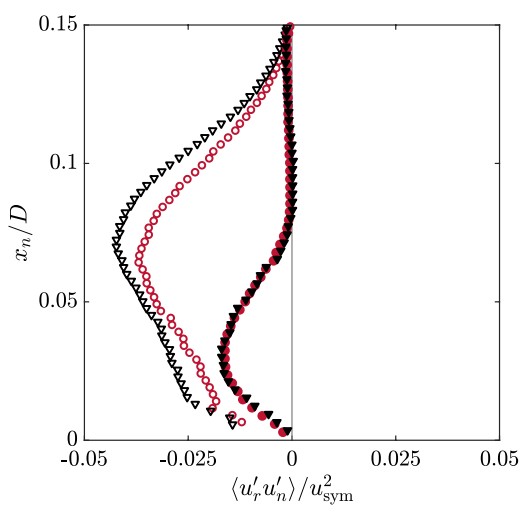

(d) At TKE-foot

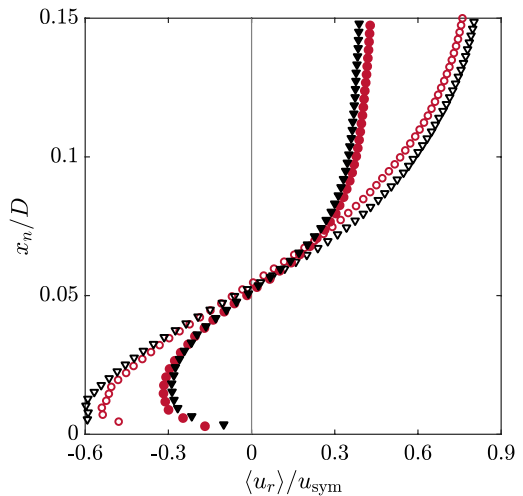

(b) At horseshoe vortex V1

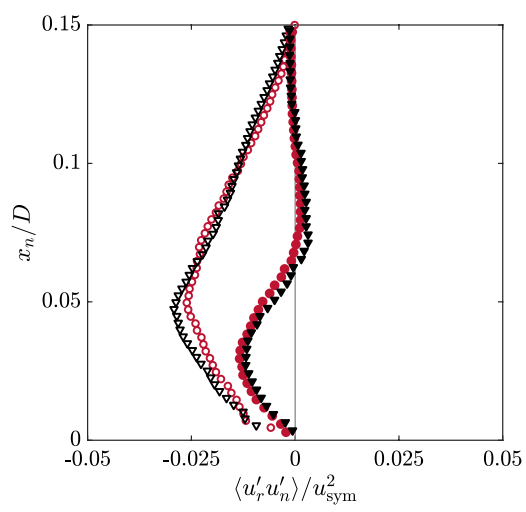

(e) At horseshoe vortex V1

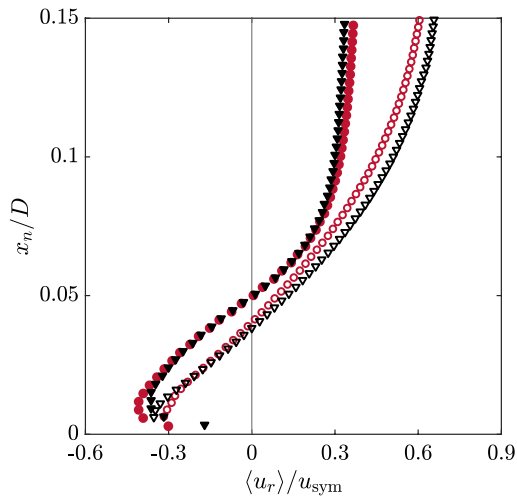

(c) Between cylinder and V1

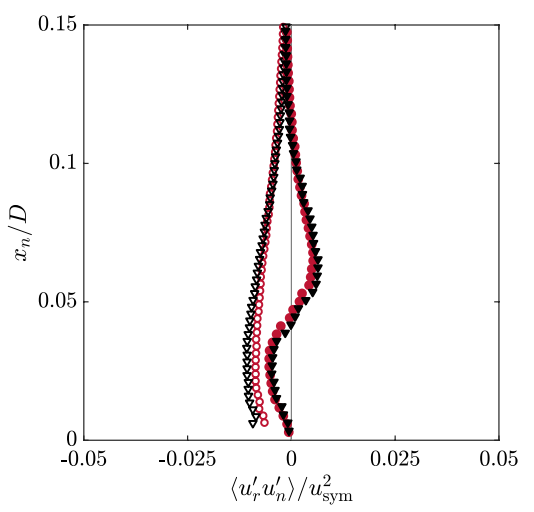

(f) Between cylinder and V1
Fig. 12 Time-averaged wall-normal profiles in the symmetry plane $\left(0^{\circ}\right)$ of the wall-parallel velocity component $\left\langle u_{r}\right\rangle / u_{\text {sym }}(\mathbf{a}-\mathbf{c})$, and $\left\langle u_{r}^{\prime} u_{n}^{\prime}\right\rangle / u_{\text {sym }}^{2}(\mathbf{d}-\mathbf{f})$ for $\operatorname{Re}=20,000$ and $\operatorname{Re}=39,000$. Hollow sym-

the $x$-coordinate was scaled (Eq. 2), such that the profile between the cylinder and V1 was taken at a comparable location for all flow configurations:

$x_{\mathrm{adj}}=\frac{x / D-x_{\mathrm{S} 3}}{x_{\mathrm{S} 3}-x_{\mathrm{V} 1}}$.

The down-flow along the cylinder front is deflected at the scour surface at S3 and a wall-parallel accelerating jet develops. In the scour, this near-wall flow appears to be similar to that in the flat-bed case, i.e., the flow accelerates from $\mathrm{S} 3$ onwards in the uphill direction until V1 and decelerates after having passed under the horseshoe vortex (Fig. 12a-c). However, the jet seems to be initially thinner and is growing faster in thickness in the scour hole than on a flat plate. The distance of the vortex centre increases marginally from $0.05 D$ in the flat-bed case to $0.052 D$ in the scour (Fig. 12b). The peak velocity of the near-wall jet grows to larger values in the scour hole reaching approximately $-0.6 u_{\text {sym }}$ underneath the horseshoe vortex centre, which is about twice as large as in the flat-bed case. Furthermore, the location of the bols refer to present scour data; solid symbols refer to data of Jenssen (2019) in the flat-bed case

peak velocity is closer to the wall than on the flat plate and in both cases not in the middle between the vortex centre and the wall. While the peak of the jet seems to be well resolved by the measurement, its evolution towards the wall is not well captured. Based on the observations that (i) the velocity peak of the jet is larger and (ii) the location of the peak is closer to the wall, we expect that the wall-shear stress is larger in the scour hole than on the flat plate.

Like in the wall jet, the flow above the horseshoe vortex reaches significantly larger values in the scour than on the flat plate. As a consequence, the velocity gradient $\partial\left\langle u_{\mathrm{r}}\right\rangle / \partial x_{n}$ increases along this profile, as well. This goes in line with the larger vorticity of the horseshoe vortex observed in the scour hole.

The Reynolds shear stresses $\left\langle u_{r}^{\prime} u_{n}^{\prime}\right\rangle$ (Fig. 12d-f) are small between the cylinder and V1 but large above the TKE foot. These large values can be explained by the large production rates in this region (compare Fig. 10). There is no change in sign below the velocity peak of the wall jet which indicates that the near-wall velocity profile of the wall jet cannot be expected to follow the turbulent law of 
the wall. Furthermore, the sign and level of the Reynolds shear stress above the velocity peak of the wall jet will not correlate with the wall-shear stress and will not be usable for wall-shear stress estimations.

\subsection{Wall-shear stress}

In what follows, we give an estimation of the wall-shear stress. In contrast to other authors, we do not use estimations based on the Reynolds shear stress as it is small and counter gradient in the wall jet. Consequently, we estimate the wall-shear stress by the velocity gradient between the wall and the first measured value. Since we could not well resolve the near-wall profile, this method can deliver a lower limit to the wall-shear stress only.

The friction coefficient $c_{\mathrm{f}}=\tau_{\mathrm{w}} /\left(\rho / 2 \cdot u_{\mathrm{sym}}^{2}\right)$ is shown in Fig. 13. The corresponding values of the approaching boundary layer are $c_{\mathrm{f}, 0}=4.62 \times 10^{-3}$ and $c_{\mathrm{f}, 0}=4.09 \times 10^{-3}$ for $\operatorname{Re}=20,000$ and $\operatorname{Re}=39,000$, respectively. In both Reynolds numbers, we observe an increase of the wallshear stress under the horseshoe vortex with respect to the approaching flow.

In the symmetry plane, the down-flow impinges at the scour surface at the stagnation point $\mathrm{S} 3$, which is indicated by zero wall-shear stress at $x_{r} / D \approx-0.6$. Towards the cylinder, the wall-shear stress becomes positive, which is a hint for a small corner vortex V3 similar to the flat-bed case (Jenssen 2019). In the uphill direction, the values of $c_{\mathrm{f}}$ become negative due to the direction of the near-wall jet. The wall-shear stress increases along the scour surface until a negative peak is reached under the horseshoe vortex where the friction coefficient $c_{\mathrm{f}}$ at $\mathrm{Re}=20,000$ is approximately twice as large as for $\operatorname{Re}=39,000$. Further uphill, the wallshear stress rapidly decreases towards zero. At $x_{r} / D \approx-1.1$, a secondary local (negative) peak occurs, which we attribute to a change in slope in the scour surface at this position.

At $45^{\circ}$, the azimuthal velocity component is included in the wall-parallel velocity component to estimate the gradient and its magnitude is documented in Fig. 13b. Note that the negative sign accounts for the local uphill flow direction. The measured near-wall values of the azimuthal component are quite noisy due to a limited number of statistical samples obtained for the out-of-plane component. At $\mathrm{Re}=39,000$, we see approximately the distribution of the wall-shear stress as observed in the symmetry plane: the wall-shear stress increases from the cylinder to the horseshoe vortex where it reaches its maximum. The distribution is different at $\mathrm{Re}=20,000$ reaching a maximum value at a smaller distance to the cylinder. This could be the effect of the azimuthal velocity component which is largest near the cylinder (compare Fig. 5).

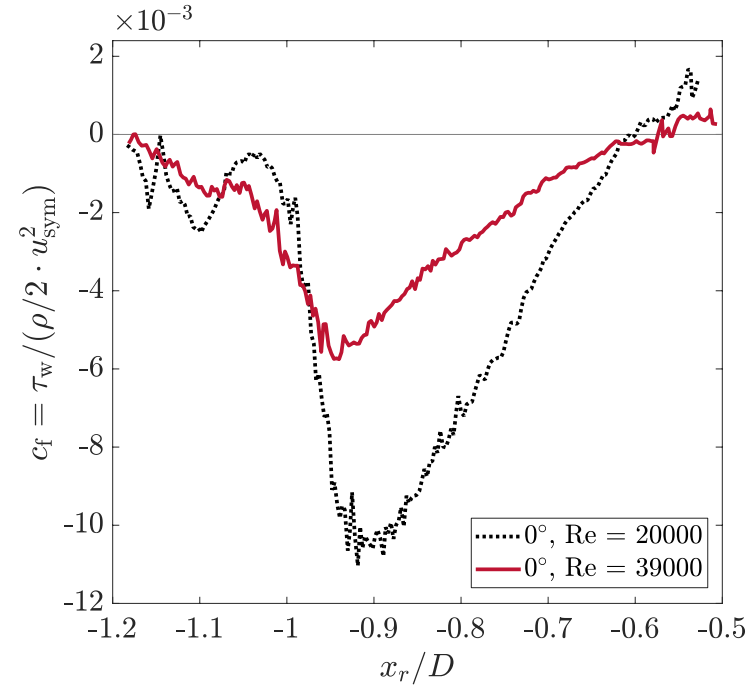

(a) $0^{\circ}$

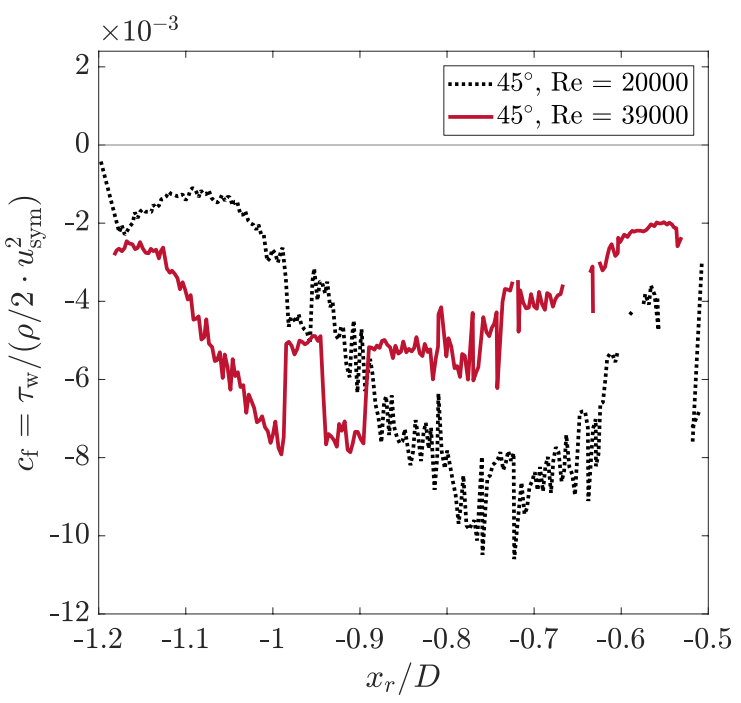

(b) $45^{\circ}$

Fig. 13 Time-averaged friction coefficient $c_{\mathrm{f}}$ in the symmetry plane (a) upstream and in a vertical azimuthal plane at $45^{\circ}$ of a scoured cylinder (b) for both Reynolds numbers

\subsection{Probability density functions of the velocity}

Devenport and Simpson (1990) detected bimodal probability density functions (PDF) of the streamwise velocity component under the horseshoe vortex in front of a wing-body junction. Since then, the bimodality of the PDFs was used to characterise the dynamics of the near-wall jet and the horseshoe vortex system by a back- and a zero-flow mode. Apsilidis et al. (2015) investigated a cylinder-wall-junction flow and postulated the occurrence of an intermediate mode being the transition from the back-flow to the zero-flow mode and vice versa. They found bimodal PDFs in considerably 
smaller regions than what was observed by Devenport and Simpson (1990). Kirkil et al. (2009) identified bimodal oscillations of the horseshoe vortex as well in their DES results in the scour. However, their results indicate bimodal velocity distributions also around the horseshoe vortex centre, which is contrasting to the observations of Devenport and Simpson (1990) and Apsilidis et al. (2015). The latter identified the bimodality in the wall-parallel jet only. An experimental confirmation of bimodal velocity distributions in a scour hole has not been undertaken so far.

Therefore, we analyse the joint probability density functions (jPDF) of the streamwise and vertical velocity components $u$ and $v$, respectively, by scatter plots in Fig. 14. We evaluate the data of $\operatorname{Re}=39,000$ normalized by $u_{\text {sym }}$. The probability isolines of the jPDF are added for $p=0.05$ to $p=0.20$. The inclination and shape of these isolines indicate the preferred alignment of the velocity fluctuations and, thus, Reynolds stresses. Moreover, we added a profile along the main axis of the jPDF shown in a miniature figure to highlight a possible bimodal distribution of the jPDF. The time-series were extracted from each sampling point and the eight surrounding grid points. We averaged these nine interrogation windows to increase the number of samples contributing to the jPDF.

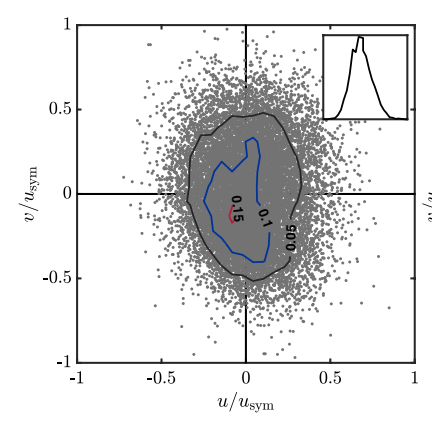

(a) Point P1

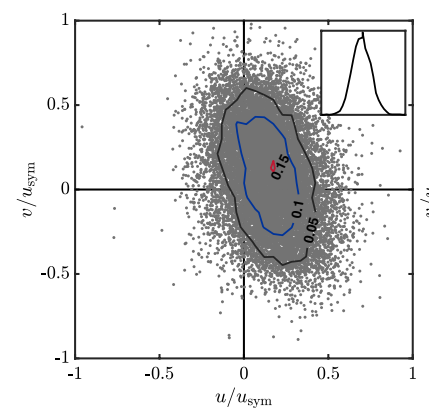

(c) Point P3

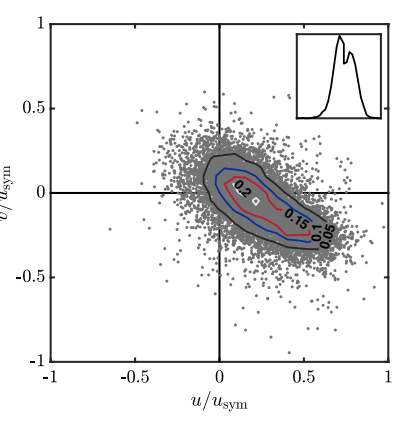

(b) Point P2

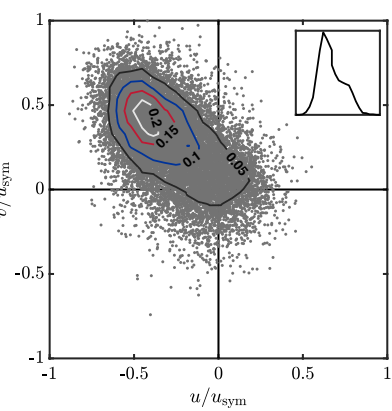

(d) Point P4
Fig. 14 Joint probability densities $u$ and $v$ in various points in the symmetry plane at $\mathrm{Re}=39,000$. P1 at the horseshoe vortex centre (a); P2 in the detached shear layer (b); P3 at the maximum TKE production (c); and P4 inside the foot of the TKE distribution (d)
The locations of these sampling points are shown in Fig. 7: (P1) at the horseshoe vortex centre (Fig. 14a); (P2) in the approaching shear layer from the scour rim (Fig. 14b); (P3) at the maximum production of TKE (Fig. 14c); and (P4) inside the foot of the c-shaped TKE distribution branching to the scour surface (Fig. 14d).

The joint probability densities (Fig. 14) support the findings from the Reynolds stresses (Figs. 8, 9). In the shear layer detaching from the rim (P2), the main axis of the fluctuations is inclined to the mean streamlines, i.e., $u^{\prime}$ and $v^{\prime}$ are negatively correlated in the Cartesian coordinate system which gives large values of $\left\langle u^{\prime} v^{\prime}\right\rangle$. The scour-oriented system is nearly aligned with the main axis of the jPDF which gives large values of $\left\langle u_{r}^{\prime} u_{r}^{\prime}\right\rangle$ and small values of $\left\langle u_{n}^{\prime} u_{n}^{\prime}\right\rangle$ as a consequence. Following the streamlines, the main axis of the jPDF turns towards the vertical axis at the maximum TKE production (P3), while it becomes vertical in the horseshoe vortex centre (P1). In the foot of the TKE (P4), the fluctuations are predominantly wall-parallel, i.e., aligned by approximately $35^{\circ}$ with respect to the $x$-axis according to the local scour inclination.

Another observation in Fig. 14 is that none of the joint probability densities reveal a clear bimodal structure, and therefore, we cannot confirm the findings of Kirkil et al. (2009), who identified bimodal PDFs in a wide region upstream and downstream of the horseshoe vortex centre. Point P2 (being in the detached shear layer from the rim) shows two peaks which, however, lie closely together. Point P4 (being in the TKE foot) shows a PDF that has a main peak at large negative $u$-velocities and a shoulder around $u=0$. This shape of a PDF is similar to what was reported by Devenport and Simpson (1990) below the horseshoe vortex centre above the bimodal region and consistent with the occurrence of a back-flow and zero-flow modes described herein. Therefore, we have a closer look at the characteristics of the near-wall flow by analysing the PDFs of the wallparallel velocity component $u_{r}$ in the next paragraph.

Doing so, an additional sampling point P5 was selected, which is located underneath $\mathrm{P} 4$ closer to the scour surface in a wall-normal sense. The corresponding PDFs from the symmetry plane as well as at $45^{\circ}$ are shown in Fig. 15. The point $\mathrm{P} 4$ lies at the maximum of the wall-parallel velocity fluctuations and its location varies, therefore, between the symmetry plane and $45^{\circ}$. The wall-normal distances of P4 and P5 in the symmetry plane are $x_{n} / D=0.0111$ and $x_{n} / D=0.0064$, respectively. Whereas in the lateral plane at $45^{\circ}$, the points $\mathrm{P} 4$ and P5 have a wall-normal distance of $x_{n} / D=0.02$ and $x_{n} / D=0.013$. These distributions show that (i) our measurement technique is able to detect bimodal behaviour in general if present; and (ii) the near-wall flow reveals two distinct modes, which are more pronounced at $\operatorname{Re}=20,000$ and in the lateral measurement plane. The first mode exceeding the time-averaged value 


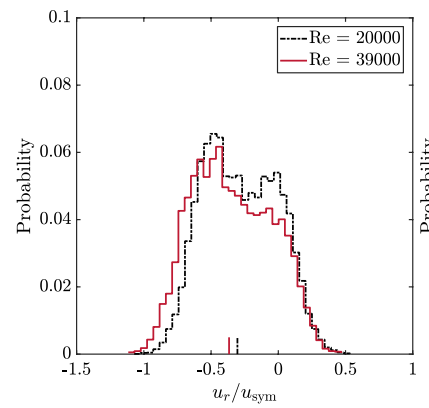

(a) $\mathrm{P} 4: 0^{\circ}, x_{n} / D=0.0111$

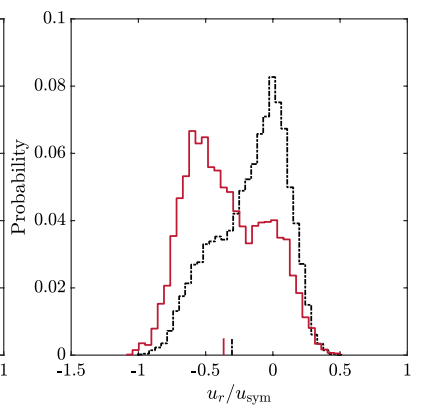

(b) $\mathrm{P} 5: 0^{\circ}, x_{n} / D=0.0064$

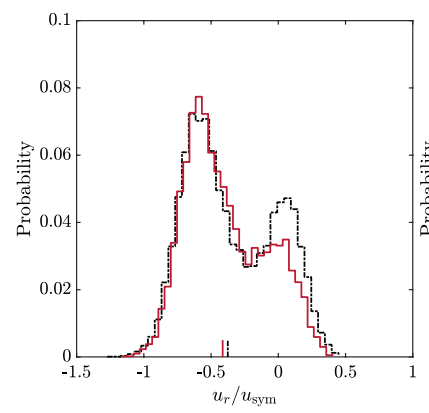

(c) $\mathrm{P} 4: 45^{\circ}, x_{n} / D=0.02$

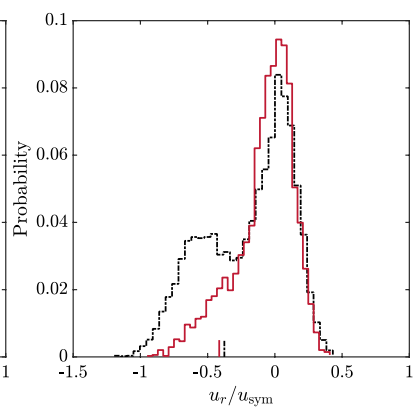

(d) $\mathrm{P} 5: 45^{\circ}, x_{n} / D=0.013$

Fig. 15 Probability density function of the wall-parallel velocity component $u_{r}$ at the locations P4 (left column) and P5 (right column) in the symmetry plane $0^{\circ}$ (top row) and at a vertical azimuthal plane at $45^{\circ}$ (bottom row) for $\operatorname{Re}=20,000$ and $\operatorname{Re}=39,000$. Ticks indicate the time-averaged values

in the negative direction could be attributed to a back-flow mode, while the second at $u_{r} \approx 0$ could be attributed to a zero-flow mode. Devenport and Simpson (1990) showed the PDFs of the streamwise velocity component along a vertical profile through the horseshoe vortex centre. With increasing distance to the bottom wall, the bimodal distributions shifted from the zero-flow mode towards the backflow mode until they vanished approximately at the height of the vortex core. We can clearly observe a similar shift of the probability maximum from $u_{r}=0$ to $u_{r} \approx-0.5 u_{\text {sym }}$ when moving away from the wall, i.e., from P5 to P4 for $\mathrm{Re}=20,000$ in the symmetry plane and for both Reynolds numbers at $45^{\circ}$. The PDF at P5 in the symmetry plane at $\mathrm{Re}=39,000$ is similar to the one at P4 at the lower Reynolds number. This could be explained by a thinner wall layer at the higher Reynolds number as we are at the limit of our spatial resolution in the wall layer.

The PDFs at P4 and P5 demonstrate that very large velocity magnitudes can occur very close to the wall. While the time-averaged values are at about $-0.4 u_{\text {sym }}$, rare events with up to $-1.0 u_{\text {sym }}$ occur. This means that the instantaneous wall-shear stress is likely to reach twice the values than the time-averaged ones presented in Fig. 13.

\section{Summary and conclusion}

We performed stereo-PIV measurements of the flow inside a scour hole at two different Reynolds numbers: $\mathrm{Re}=$ 20,000 and $\operatorname{Re}=39,000$. The scour hole represents an intermediate state with a depth of approximately one half of the cylinder diameter. It was milled from aluminium and coated by a fluorescent varnish to reduce surface reflections of the light sheet. We took a special care to obtain a sufficient number of samples to compute the first- and second-order statistics. The measurements were conducted at two vertical planes around the cylinder: in the symmetry plane and rotated by $45^{\circ}$ in the azimuthal direction. To our knowledge, the measurements are the first non-intrusive, high-resolution measurements of all three components of the velocity vector in a scour hole around an entire cylinder so far.

The down-flow in front of the cylinder and the shear layer detaching from the scour rim drive the vortex system in the scour hole. These findings are in line to what previous authors published.

In the time-averaged flow field, we find a single strong and large horseshoe vortex. This is different to previous publications, some of which reported a foot vortex at the cylinder-wall junction and some others reported a secondary vortex system between the rim and the main horseshoe vortex. The position of the horseshoe vortex is in line with published LES and DES results, but departs from most of the published experimental results. Our measurements seem to be the first to corroborate the LES/DES results of Kirkil et al. (2008) and Kirkil et al. (2009) in this aspect. We measured vorticity values which are larger than what was observed before (experimentally and numerically). We attribute the reason for this to the spatial resolution which was considerably higher in our experiments than in other studies. The vorticity in the horseshoe vortex centre and the back-flow velocity underneath are nearly independent on Reynolds number and considerably larger than what was found in the flat-plate case. Three factors contribute to this observation: (i) the larger space which is available for the horseshoe vortex in the scour hole; (ii) the larger momentum in the down-flow; and (iii) the larger vorticity values in the fluid which is drawn into the centre of the vortex.

We found enlarged levels of turbulent kinetic energy in the shear layer detaching from the rim connected to the area around the horseshoe vortex. Furthermore, there is a foot at the wall similar to the c-shaped distribution found in the flat-plate case. Being largely different from other experimentally measured distributions of turbulent kinetic energy, our results can be regarded as the first qualitative confirmation of the numerical results by Kirkil et al. 
(2008) and Kirkil et al. (2009). However, our measured levels are somewhat smaller than those. The turbulent fluctuations are inclined to the time-averaged streamlines in the shear layer separating from the rim and turn towards mainly vertical fluctuations in the horseshoe vortex centre. Near the wall under the horseshoe vortex, the fluctuations are mainly wall-parallel. This is the second peak of turbulent kinetic energy or the foot of the c-shaped distribution. The production of turbulent kinetic energy is maximal upstream of the horseshoe vortex centre with considerable contributions in the shear layer and in the foot of the turbulent kinetic energy distribution.

We observed bimodal fluctuations in a very thin layer under the horseshoe vortex centre within the area of large wall-parallel fluctuation levels. In other regions, we cannot confirm with certainty such bimodal distributions.

Turbulent momentum transport towards the wall is actuated by turbulent shear stresses in the wall-oriented coordinate system. These shear stresses are (i) nearly Reynolds number independent; (ii) large at the horseshoe vortex centre and uphill of it; (iii) small downhill of the horseshoe vortex centre (closer to the cylinder); and (iv) small and counter gradient in the wall jet between its peak velocity and the wall. From the latter observation, we conclude that models for the wall-shear stress estimation either based on the law of the wall for turbulent flows or based on Reynolds stresses at larger wall distances would fail.

We have estimated the wall-shear stresses by the wallnormal velocity gradient between the first measured off-wall grid point and the wall. This is a lower bound (due to the curvature of the velocity profile). The corresponding values based on the depth-averaged velocity in the symmetry plane of the channel are larger than in the flat-bed case. Moreover, the maximum wall-shear stress is found under the horseshoe vortex centre instead inside the wall-parallel jet as in the flatbed case. At $45^{\circ}$, the wall-shear stress is presumably larger than in the symmetry plane, but there are large uncertainties in our estimation.

Acknowledgements The authors gratefully acknowledge the financial support by the German Research Association (DFG) under Grant no. MA2062/11. We would also like to thank Prof. Christian Kähler for his helpful advice while designing the stereo-PIV experiment.

Funding Open Access funding enabled and organized by Projekt DEAL.

Open Access This article is licensed under a Creative Commons Attribution 4.0 International License, which permits use, sharing, adaptation, distribution and reproduction in any medium or format, as long as you give appropriate credit to the original author(s) and the source, provide a link to the Creative Commons licence, and indicate if changes were made. The images or other third party material in this article are included in the article's Creative Commons licence, unless indicated otherwise in a credit line to the material. If material is not included in the article's Creative Commons licence and your intended use is not permitted by statutory regulation or exceeds the permitted use, you will need to obtain permission directly from the copyright holder. To view a copy of this licence, visit http://creativecommons.org/licenses/by/4.0/.

\section{References}

Adrian RJ, Westerweel J (2011) Particle image velocimetry. Cambridge University Press, Cambridge

Apsilidis N, Diplas P, Dancey CL, Bouratsis P (2015) Time-resolved flow dynamics and Reynolds number effects at a wall-cylinder junction. J Fluid Mech 776:475-511

Baker CJ (1980) The turbulent horseshoe vortex. J Wind Eng Ind Aerodyn 6:9-23

Counihan J (1969) An improved method of simulating an atmospheric boundary layer in a wind tunnel. Atmos Environ 3:197-214

Dargahi B (1990) Controlling mechanism of local scouring. J Hydraul Eng 116(10):1197-1214

Das S, Das R, Mazumdar A (2013) Circulation characteristics of horseshoe vortex in scour region around circular piers. Water Sci Eng 6(1):59-77

Devenport WJ, Simpson RL (1990) Time-dependent and time-averaged turbulence structure near the nose of a wing-body junction. J Fluid Mech 210:23-55

Dey S, Raikar RV (2007) Characteristics of horseshoe vortex in developing scour holes at piers. J Hydraul Eng 133(4):399-413

Fernholz HH, Finley PJ (1996) The incompressible zero-pressure-gradient turbulent boundary layer: an assessment of the data. Prog Aerosp Sci 32:245-311

Gobert C, Link O, Manhart M, Zanke U (2009) Discussion of coherent structures in the flow field around a circular cylinder with scour hole by G. Kirkil, S. G. Constaninescu and R. Ettema. J Hydraul Eng 136(1):82-4

Graf W, Istiarto I (2002) Flow pattern in the scour hole around a cylinder. J Hydraul Res 40(1):13-20

Jenssen U (2019) Experimental study of the flow around a scouring bridge pier. $\mathrm{PhD}$ thesis, Technische Universität München

Khosronejad A, Kang S, Sotiropoulos F (2012) Experimental and computational investigation of local scour around bridge piers. Adv Water Resour 37:73-85

Kirkil G, Constantinescu G (2010) Flow and turbulence structure around an in-stream rectangular cylinder with scour hole. Water Resour Res 46(11)

Kirkil G, Constantinescu G, Ettema R (2008) Coherent structures in the flow field around a circular cylinder with scour hole. J Hydraul Eng 134(5):572-587

Kirkil G, Constantinescu G, Ettema R (2009) Detached Eddy simulation investigation of turbulence at a circular pier with scour hole. J Hydraul Eng 135(11):888-901

Kirkil G, Constantinescu S, Ettema R (2010) Closure to of "Coherent structures in the flow field around a circular cylinder with scour hole" by G. Kirkil, S.G. Constantinescu, and R. Ettema. J Hydraul Eng 136(1):84-86

Link O, Pfleger F, Zanke U (2008) Characteristics of developping scour-holes at sand-embedded cylinder. Int J Sedim Res 23:258-266

Melville B (1975) Local scour at bridge sites. PhD thesis, University of Auckland

Melville BW (2008) The physics of local scour at bridge piers. In: Fourth international conference on scour and erosion

Melville BW, Raudkivi AJ (1977) Flow characteristics in local scour at bridge piers. J Hydraul Res 15(4):373-380 
Paik J, Escauriaza C, Sotiropoulos F (2007) On the bimodal dynamics of the turbulent horseshoe vortex system in a wing-body junction. Phys Fluids 19:045107

Pfleger F (2011) Experimentelle Untersuchung der Auskolkung um einen zylindrischen Brückenpfeiler. PhD thesis, Technische Universität München

Roulund A, Mutlu Sumer B, Fredsoe J, Michelsen J (2005) Numerical and experimental investigation of flow and scour around a circular pile. J Fluid Mech 534:351-401

Schanderl W, Manhart M (2016) Reliability of wall shear stress estimations of the flow around a wall-mounted cylinder. Comput Fluids 128:16-29

Schanderl W, Jenssen U, Strobl C, Manhart M (2017) The structure and budget of turbulent kinetic energy in front of a wall-mounted cylinder. J Fluid Mech 827:285-321
Sciacchitano A, Scarano F (2014) Elimination of PIV light reflections via a temporal high pass filter. Meas Sci Technol 25(8):084009

Theunissen R, Scarano F, Riethmuller M (2008) On improvement of PIV images near stationary interfaces. Exp Fluids 45(4):557-572

Unger J (2006) Strömungscharakteristika um kreiszylindrische Brückenpfeiler. PhD thesis, Eidgenössische Technische Hochschule Zürich

Unger J, Hager WH (2007) Down-flow and horseshoe vortex characteristics of sediment embedded bridge piers. Exp Fluids 42(1):1-19

Publisher's Note Springer Nature remains neutral with regard to jurisdictional claims in published maps and institutional affiliations. 